\title{
A PET Study of Visuospatial Attention
}

\author{
Maurizio Corbetta, Francis M. Miezin, Gordon L. Shulman, and Steven E. Petersen \\ Department of Neurology and Neurological Surgery, and the McDonnell Center for the Study of Higher Brain Function, \\ Washington University School of Medicine, St. Louis, Missouri 63110
}

\begin{abstract}
Positron emission tomography (PET) was used to identify the neural systems involved in shifting spatial attention to visual stimuli in the left or right visual field along foveofugal or foveocentric directions. Psychophysical evidence indicated that stimuli at validly cued locations were responded to faster than stimuli at invalidly cued locations. Reaction times to invalid probes were faster when they were presented in the same than in the opposite direction of an ongoing attention movement. PET evidence indicated that superior parietal and superior frontal cortex were more active when attention was shifted to peripheral locations than when maintained at the center of gaze. Both regions encoded the visual field and not the direction of an attention shift. In the right superior parietal lobe, two distinct responses were localized for attention to left and right visual field. Finally, the superior parietal region was active when peripheral locations were selected on the basis of cognitive or sensory cues independent of the execution of an overt response. The frontal region was active only when responses were made to stimuli at selected peripheral locations. These findings indicate that parietal and frontal regions control different aspects of spatial selection. The functional asymmetry in superior parietal cortex may be relevant for the pathophysiology of unilateral neglect.
\end{abstract}

IKey words: spatial attention, positron emission tomography (PET), visual information processing, frontal cortex, parietal cortex, unilateral neglect]

A typical visual scene is composed of many different objects, but we "pay attention" to only a few of them at any given time. Shifting attention to objects in the visual field is a component of many perceptual-motor processes, since spatial selection may be involved in detecting (Posner, 1980) or identifying (Eriksen and Hoffman, 1972) objects, or executing a response to an object such as an cyc (Rcmington, 1980; Shepherd et al., 1986) or hand movement.

Spatial selection is often controlled in psychological experiments by a cueing procedure that manipulates the probability

\footnotetext{
Received May 12, 1992; revised Aug. 21, 1992; accepted Sept. 25, 1992.

We thank Elizabeth Raife for computer programming, Antonella Rastelli for drawing Figure 4, and Paula Jost for editorial assistance. We also thank Giovanni Berlucchi, Carol Colby, Mike Posner, and Marcus Raichle for helpful comments on a first version of the manuscript. This work was supported by NIH Grants NS06833, NS25233, and EY08775, Office of Naval Research Grant N00014-89$\mathrm{J}-1426$, and the McDonnell Center for the Study of Higher Brain Function.

Correspondence should be addressed to Maurizio Corbetta, Department of Neurology and Neurological Surgery, Washington University School of Medicine, Box 8111 , St. Louis, MO 63110.

Copyright (C) 1993 Society for Neuroscience $0270-6474 / 93 / 131202-25 \$ 05.00 / 0$
}

of appearance of a target at a particular location. Cognitive studies in human and nonhuman primates have demonstrated that visual performance on a variety of detection, discrimination, and identification tasks is improved (benefits) or decreased (costs) by correct or incorrect cueing of target location (Eriksen and Hoffman, 1972; Petersen et al., 1987; Downing, 1988). Costs and benefits in these paradigms are independent of eye movements (Posner, 1980), and have a spatiotemporal distribution that can be accurately measured (Eriksen and Hoffman, 1972; Posner, 1980; Posner and Cohen, 1984; Maylor, 1985; Shulman et al., 1985; Berlucchi et al., 1989; Muller and Rabbitt, 1989). The effects of spatial cueing on processing are thought to reflect the action of spatial selection mechanism(s) for shifting the focus of processing to cued objects or locations.

Regions of the parietal and frontal lobe that are reciprocally connected (Jones and Powell, 1970; Petrides and Pandya, 1984; Matelli et al., 1986; Selemon and Goldman-Rakic, 1988; Andersen et al., 1990a) might be important for implementing spatial attention operations. Lesions in inferior parietal and/or frontal (dorsolateral and anterior cingulate) cortex cause an inability to attend and respond to contralateral stimuli in both human and nonhuman primates (unilateral neglect syndrome; for reviews, see DeRenzi, 1982; Heilman et al., 1987b; Milner, 1987; Rizzolatti and Camarda, 1987). Although unilateral neglect is a complex syndrome involving a variety of deficits, some features have been related to a deficit in controlling components of visual atlention as defined by spatial cueing procedures (Posner et al., 1982, 1984). ERP recordings in normal volunteers show that spatial selection of a target location modulates specific components of the evoked potential that have been localized to posterior brain regions (Harter et al., 1982; Hillyard and Picton, 1987; Mangun et al., 1987). Single-unit recording studies in macaques indicate that neurons in posterior parietal cortex (Bushnell et al., 1981) and the interconnected lateral pulvinar nucleus of the thalamus (Petersen et al., 1985) show a spatially selective enhancement of a visual response when the animal is covertly attending to a stimulus location. Neurons in frontal eye fields (Bushnell et al., 1981; Bruce and Goldberg, 1985), dorsolateral prefrontal cortex (Boch and Goldberg, 1989; Funahashi et al., 1991), caudate nucleus (Hikosaka et al., 1989), and superior colliculus (Wurtz and Goldberg, 1972; Wurtz and Mohler, 1976), in contrast, show a selective enhancement only before the execution of an eye movement to the stimulus location.

The distributed nature of these effects raises the problem of specifying the relative contribution of each region to the control of spatial selection. Introspectively, attention may be shifted to an object because its physical characteristics differ from those of the surrounding background, or because that particular object matches our current expectations or goals. While walking along 
a uniformly gray sidewalk, one may spot a bright yellow tennis ball "popping out" of the background; attention is mostly driven by a strong figure-ground segregation signal. When first looking at the Mona Lisa, one may search the mouth region for her famous smile; attention is shifted on the basis of previous knowledge about that particular painting. These two cases correspond to the psychological distinction between spatial selection generated via exogenous (sensory) or endogenous (cognilive) cues (Jonides, 1981; Muller and Rabbitt, 1989) and may involve different neural pathways.

The distinction between perception and intention (Mesulam, 1981; Heilman et al., 1987a; Bisiach et al., 1990), based on studies of cortical lesions and unilateral neglect, may also account for the involvement of different neural regions in spatial selection. Some deficits, such as the inability to detect a sensory stimulus on the contralesional side when a second stimulus is simultaneously presented on the ipsilesional side (extinction), or the inability to describe the left side of an imaged visual scene (Bisiach et al., 1981), seem "perceptual" in nature. Other deficits, such as the difficulty to initiate and/or perform a movement with either arm toward the contralesional side of space, independent of the side of stimulus presentation (Heilman et al., 1985; Coslett et al., 1990), seem "premotor," or response, related. Different neural regions may mediate the spatial selection involved in perceptual and intentional processing.

A final issue relevant to the present study is the internal organization of these distributed brain representations. Lesion analyses of unilateral neglect patients have suggested that visual attention may be mapped by side, either hemifield or hemispace (Mesulam, 1981; DeRenzi, 1982; Calvanio et al., 1987; Heilman et al., 1987b; Ladavas, 1987; Farah et al., 1990), or by direction (Kinsbourne, 1977; Posner et al., 1987a; Reuter-Lorenz and Posner, 1990). If attention is coding visual field, right (left) hemisphere regions should be mostly active for selection of left (right) side stimuli, irrespective of whether attention is shifting in a leftward or rightward direction. Alternatively, if attention is coding direction, right (left) hemisphere regions should be mostly active for leftward (rightward) shifts of spatial attention, irrespective of whether the left or right visual field is selected.

The main goal of the present set of experiments is to localize regions of the human brain that are related to shifts of visuospatial attention. Regional blood flow changes were measured with positron emission tomography (PET) in normal volunteers during a series of psychophysical tasks in which different field locations were spatially cued. Several task conditions were run in the same individual to isolate effects related to both exogenous and endogenous, and/or perceptual and premotor, components of spatial selection. The importance of the visual field and/or the direction of attention in controlling the pattern of regional brain activation was studied by factorially crossing these two variables.

\section{Materials and Methods}

\section{Subjects}

Subjects were normal volunteers drawn from the population of students, residents, and fellows in the medical, allied health, and graduate schools of Washington University. All were strongly right handed as assessed by the Edinburgh handedness inventory. Volunteers ranged from 18 to 28 years of age and all reported normal or corrected to normal visual acuity. Informed consent was obtained following guidelines approved for this study by the Human Studies Committee, and the Radioactive Drug Research Committee of Washington University. Twenty-four volunteers (19 women, 5 men) were tested in the main PET/psychophysical experiment, and a different group of six volunteers ( 3 men, 3 women) was studied in a pilot psychophysical experiment. Volunteers for the PET experiment were screened on the basis of their psychophysical results in the main experimental task (see below).

\section{Apparatus}

Subjects were tested in a blackened sound-proof room during session 1 , in which psychophysical data were collected, and in the scanner room during session 2, in which psychophysical and PET data were collected. During session 2, each subject lay on a scanner couch and wore an individually molded, closely fitted, plastic facial mask to ensure head stability (Fox et al., 1985a). The room was dimly illuminated and equipment cooling fans produced a low-level background noise. Visual stimuli were generated using a Macintosh II system and displayed on an RGB monitor positioned $42 \mathrm{~cm}$ from the subject, subtending a visual angle of $32^{\circ}$. Eye movements were monitored using electro-oculography (EOG). Accuracy and reaction times (RTs) were collected with a microswitch connected to the Macintosh II system.

\section{Psychophysical procedures}

The visual display consisted of a horizontal row of 10 small box outlines whose centers were positioned $2^{\circ}$ in the lower visual field at $1^{\circ}, 3^{\circ}, 6^{\circ}$, $10^{\circ}$, and $15^{\circ}$ of visual angle in the left and right hemifield (Fig. $1 \mathrm{~A}$ ). A small fixation cross hair was centered within an 11 th box outline in the center of the display. The size of each box was $1^{\circ} \times 1^{\circ}$ of visual angle, and each arm of the fixation cross hair was $0.25^{\circ}$ of visual angle. All line segments in the the display were white on a black background with a luminance of $2.6 \mathrm{~cd} / \mathrm{m}^{2}$. The probe stimulus that subjects detected in the various tasks was an asterisk filling a box for $150 \mathrm{msec}$. In both sessions subjects were tested with various detection tasks designed to manipulate spatial attention in different ways.

Shifting attention task. This task was designed to induce endogenous (cognitive) shifts of attention to peripheral locations within a visual field and along a particular direction. On $80 \%$ of the probe presentations, the probe stimulus appeared at a predictable location according to a previously instructed leftward or rightward sequence of stimulation (valid trials). For instance, during a right visual field-right direction (RVF/ RD) condition, the block started with the presentation of a static stimulus in the $1^{\circ}$ location of the right visual field. When ready, the subject pressed the key to blank the stimulus. After a random delay (see below) the first probe appeared at $3^{\circ}$ with an $80 \%$ probability, after another random delay the second probe appeared at $6^{\circ}$ with the same probability, and so on. When the most peripheral location $\left(15^{\circ}\right)$ was stimulated, the sequence restarted from the most central location for the next cycle (Fig. $1 B)$. Right visual field locations were therefore stimulated mostly following a right direction. On the remaining $20 \%$ of the probe appearances, unpredictable positions were probed (invalid trials). In this case the predictive sequence was restarted from the location next to the one just probed with a probability of $80 \%$. Similarly, a right visual field-left direction $(\mathrm{RVF} / \mathrm{LD})$ condition started with the presentation of a static stimulus in the $15^{\circ}$ location, the first probed location was $10^{\circ}$, the second probed location was $6^{\circ}$, and so on. The delay between probe onsets was randomly selected on each trial from three intervals $(1000,1500,2000$ $\mathrm{msec}$ ), and averaged $1500 \mathrm{msec}$. Each block was 100 trials long, yielding 80 valid ( 16 per stimulus location) and 20 invalid trials ( 4 per stimulus location). The direction of the stimulus sequence (left, right) and visual field (left, right) were factorially varied in a blocked design. Subjects were informed about the probability manipulation and spatial predictability of the probe. They were explicitly instructed to shift attention on each trial to the most probable location (without moving their eyes), and detect the stimulus onset as quickly as possible with a left hand key press. The efficacy of the attentional instruction was monitored by contrasting the reaction times (RTs) for valid trials with RTs for invalid trials. Since the preliminary pilot study had shown that valid trials averaged about $30-40 \mathrm{msec}$ faster than invalid trials, the subjects for the PET experiment were screened in session 1 to minimize performance variability and maximize the likelihood that obtained activations related to shifts of spatial attention. Only subjects that showed an average validity effect (RT difference between valid and invalid trials) greater than 20 msec were selected for the PET experiment.

Central detection task. This task was intended to prevent orienting of attention to peripheral locations by engaging subject's attention in the center. The task was designed to match the shifting attention task in total peripheral visual stimulation, motor activity, and arousal. The 


\section{A. Visual display}

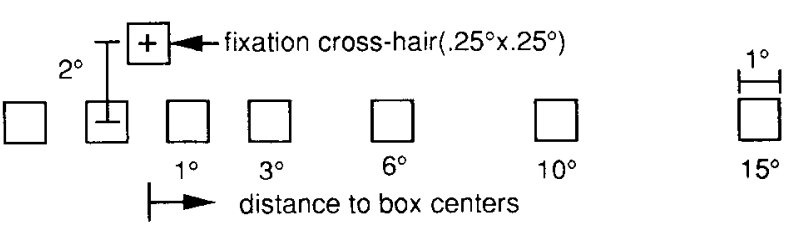

\section{B. Shifting attention RVF/RD}

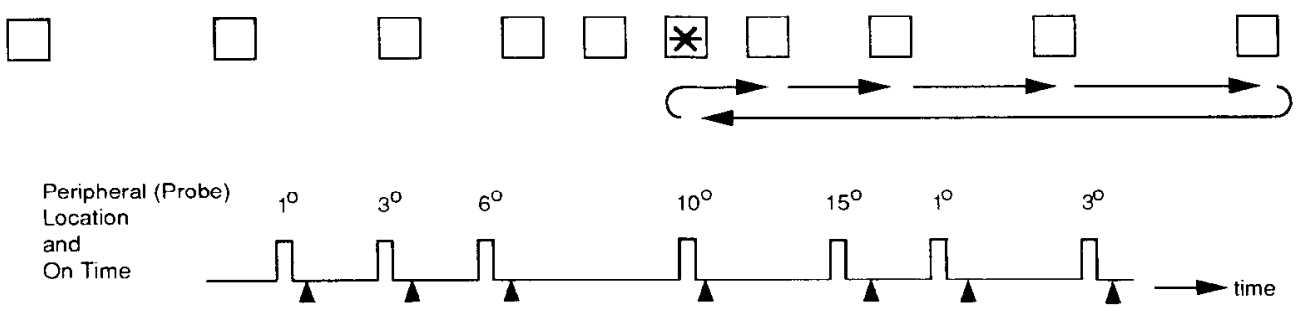

Figure 1. Visual display and task conditions (see Materials and Methods). $A$, Static visual pattern present in all tasks. $B$, Shifting attention task, right visual field/right direction condition $(R V F)$ $R D$ ). The upper part diagrams the peripheral probe stimulus (asterisk) to be detected, and its progression through the static pattern following a predictive sequence of locations (arrows). The lower part shows the temporal relationship between the presence and location of the same sequence of peripheral probes, and the subject's response. Onset time and interstimulus interval are as in the text. The two displays show only valid trials. $C$, Central detection task, right visual ficld condition. The upper part diagrams the central probe stimulus (asterisk) to be detected, superimposed on the fixation point, and the spatially random (arrows) sequence of peripheral stimuli (asterisk). The lower part indicates the temporal relationships between the presence of the peripheral stimuli and the central probes, and the subject's response. Onset time and interstimulus interval are as in the text.

\section{Central detection RVF}

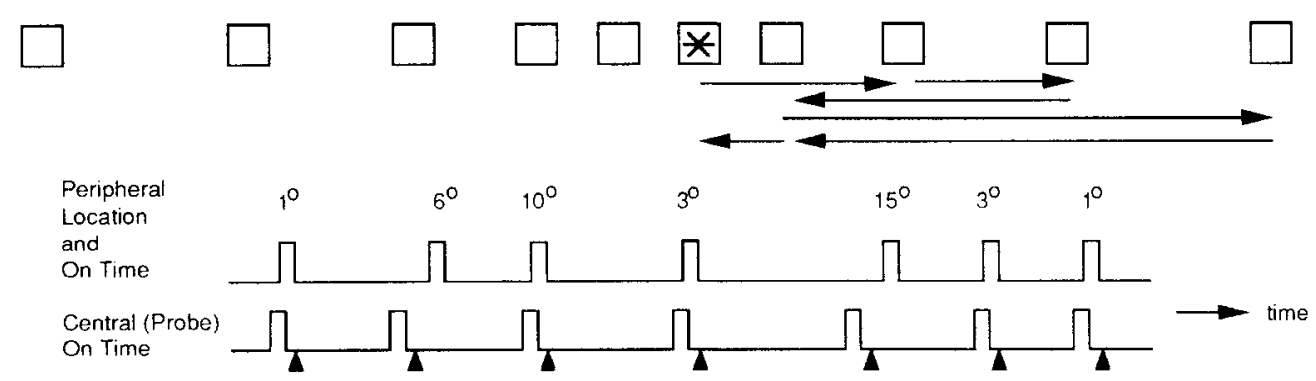

$\Delta=$ Key Press subject responded only to probe stimuli presented in the central fixation box, while peripheral stimuli (identical to those in the shifting attention condition) were flashed at random locations in either the left or right hemifield (Fig. 1C). The interval between successive central probes averaged $1500 \mathrm{msec}$ as in the shifting attention task. The peripheral stimuli were flashed randomly either 100 or $400 \mathrm{msec}$ after the central probes (these values were selected on the basis of an independent pilot study, see below). Each block was 50 trials long. Subjects were instructed to attend and respond to the central probes with a left hand key press, and to ignore the peripheral transients. Left and right visual field stimulation conditions were independently tested.

The efficacy of this task in preventing peripheral shifts of attention was evaluated in a separate study by varying the temporal relationship between central and peripheral stimuli. If subjects cannot suppress automatic orienting to the peripheral stimuli, one might expect slow RTs to the central probe when the peripheral stimulus is presented just before or simultaneously with the probe. In the pilot study, RTs to central probe stimuli were recorded in the presence or absence of spatially random peripheral stimuli (in a blocked design) that were presented at different stimulus onset asynchronies (SOAs) with respect to the central probe $(-800,-100,0,100,200,400 \mathrm{msec})$. As noted in the Results, reaction time to the central probe was independent of the SOA of the peripheral stimuli.

The central detection task differed from the shifting attention task in the presence of transient foveal stimulation (only present in the former task), the spatial pattern of peripheral stimulation (predictable in the shifting attention condition, random in the central detection task), and the degree of attention devoted to the fovea (high in the central detection task, low in the shifting attention condition).

Two other conditions were run in which no motor response was required.

Passive task. Stimuli were flashed at random peripheral locations in either the left or right hemifield with a $1500 \mathrm{msec}$ average interval between successive flashes (as in the previous two tasks). Subjects maintained fixation and did not respond to the peripheral probes. Foveal stimuli were not presented. Left and right visual field stimulation blocks were presented and each block consisted of 50 trials. The task examines the effect of peripheral visual stimulation in the absence of obvious motor activity. Since subjects were not actively engaged in a foveal detection task, the onset of transient stimuli in one visual field presumably summoned attention in an exogenous (automatic) fashion to the peripheral locations (Jonides, 1981; Muller and Rabbitt, 1989). This 
differs from the shifting attention task where subjects actively (endogenously) shifted attention to the most likely peripheral targets.

Fixation point only task. Subjects fixated on the cross hair in the central box for a period of time equivalent to 50 trials, and neither peripheral nor central stimuli were presented.

Although all tasks were completed in both sessions, sessions 1 (psychophysics only) and 2 (psychophysics/PET) differed in various respects. First, the probability of valid and invalid trials in the shifting attention tasks was slightly different in the two sessions. In session 1 the probability was the same ( $80 \%$ valid, $20 \%$ invalid) throughout a block of trials, while in session 2 the probability varied with time. The task started about $40 \mathrm{sec}$ before the intravenous injection of ${ }^{15} \mathrm{O}$-labeled water with the usual $80 \% / 20 \%$ distribution of valid and invalid trials. At injection time, the distribution of valid-invalid trials was shifted to $95 \% / 5 \%$ until the end of the scan. This period is the sum of the scanning time $(40 \mathrm{sec})$ plus a variable delay time. This delay time $(8-20 \mathrm{sec}$ in our experience) is the time between the injection and a rapid rise in brain radioactivity determined by the arrival of the tracer in the brain vasculature, which in turn triggers the onset of data collection. After the end of the scan the probability of valid-invalid trials was shifted back to the usual $80 \% / 20 \%$ for another $40 \mathrm{sec}$ period. This variation in the protocol was introduced to maximize the number of valid trials during the blood flow measurement (i.e., according to our hypothesis the number of attention shifts in a particular direction) and went undetected both subjectively and in terms of performance (see Results).

Second, during session 2 the start and end of the behavioral tasks were locked in time to the PET procedure. All tasks began about $40 \mathrm{sec}$ before injection and lasted the length of the scan (with the exception of the shifting attention task, which ran longer; see above). The actual number of trials collected on each task, therefore, was slightly different from subject to subject, and slightly different from those collected during session 1 . In the shifting attention tasks, 90 trials were collected on average in each block (range, 84-96; against 100 in session 1), yielding about 12 invalid trials. During the actual blood flow measurement, 26 trials were collected on average, yielding one or two invalid trials. For the central detection tasks, 60 trials were collected on average (range, $56-65$; against 50 in session 1), of which 26 occurred during the blood flow measurement.

Finally, the task order was different. In session 1, the four shifting attention tasks (left and right field, respectively with a lcft and right direction) were always run first (in random order across subjects). The two central detection tasks were run second (in random order across subjects), and the session ended with the two passive and fixation point only tasks. In session 2, a nine-scan series was run, one scan for each shifting attention task (four scans), one scan for each central detection task (two scans), one scan for each passive task (two scans), and one fixation point only scan. The order of scan conditions was counterbalanced across individuals according to a semirandom schedule designed to minimize movement artifacts in the relevant subtraction images. The fixation only scan was always run fifth; the passive scans were always run fourth and sixth; the central detection scans were always run second and eighth; left or right hemifield conditions were all studied respectively in the first half or second half of a scan series. A typical scan series might be (1) left visual field/left direction shifting attention (LVF/LD), (2) left visual field stimulation, central detection (LCD), (3) left visual field/right direction shifting attention (LVF/RD), (4) left visual field stimulation, passive (LPASS), (5) fixation point only (FPT), (6) right visual field stimulation, passive (RPASS), (7) right visual field/right direction shifting attention (RVF/RD), (8) right visual field stimulation, central detection (RCD), and (9) right visual field/left direction shifting attention $(\mathrm{RVF} / \mathrm{LD})$.

\section{PET scanning techniques}

The PET scanning activation methodology developed at Washington University was used. These methods are extensively described in the literature (Fox et al., 1988; Mintun et al., 1989; Petersen et al., 1989) and will be only briefly discussed here.

The subject's head was stabilized with a plastic facial mask and a lateral skull $x$-ray taken to assess head alignment in the scanner and the anatomical locations of the scan slices (Fox et al., 1985a). Water labeled with ${ }^{15} \mathrm{O}$ acting as a blood flow tracer was administered as an intravenous bolus of $8-10 \mathrm{ml}$ of saline containing $50-70 \mathrm{mCi}$. The use of ${ }^{15} \mathrm{O}$-labeled water, with its short half-life (123 sec) and short scanning time (40 sec), allowed for the performance of nine scans within an individual in a single session.

The PETT VI system was used in the low-resolution mode, simultaneously acquiring seven parallel slices with a center-to-center distance of $14.4 \mathrm{~mm}$ (Yamamoto et al., 1982). Images were reconstructed by filtered backprojection to a resolution of $17 \mathrm{~mm}$ full width half maximum and a pixel size of $2.7 \mathrm{~mm}$ by $2.7 \mathrm{~mm}$. An arterial catheter was not used and therefore the reconstructed images were not converted to blood flow values. The responses reported here are changes in radiation distribution rather than blood flow changes. Over the range tested, blood flow is very linear with distribution of radiation (Herscovitch et al., 1983). In the text, responses will be referred to as changes in blood flow.

A linear normalization was applied to reconstructed images to negate the effects of global fluctuations in activity (Fox et al., 1987). This prevented the confounding of task-induced focal changes with fluctuations affecting the entire brain (e.g., due to changes in arterial $\mathrm{pCO}_{2}$ ). For each subject, images were grouped into activation-control pairs and subtracted from each other. Images with obvious movement artifacts were excluded from further analysis (Fox et al., 1987). The resultant images are of foci of change on a baseline noise background rather than on the complex background of varying brain anatomy.

All subtraction images were transformed to a standard anatomical space (Fox et al., 1985a). Those acquired during identical behavioral states were averaged across subjects to suppress image noise and improve signal-to-noise (Fox et al., 1988).

Distribution change analysis. A maximum-detection computer algorithm (Fox et al., 1988; Mintun et al., 1989) was used to identify all positive and negative local maxima in each averaged image. A twotiered statistical analysis was applied to these sets of regional foci. First, an omnibus test $(\gamma-2$ statistic) was used to determine whether an image (a population of regional changes) had any significant responses (distribution outliers). Second, as a post hoc analysis, the magnitude of a response was described relative to the noise level by $z$ score (Fox et al., 1988), and a cutoff of $z=1.96$ was usually adopted. These techniques were originally developed for blood flow change data but have been also validated for activity change data (Fox and Mintun, 1989).

In this article the distribution change analysis will be used to assess the general significance of an image (omnibus test), and as a survey technique to describe the regions of activations in the various conditions. However, this analysis does not accurately estimate the significance of individual responses and their interindividual variability in terms of magnitude or location (see Friston et al., 1991; Worsley et al., in press, for a discussion of some of these methodological issues). Furthermore, low-level responses consistently activated across conditions tend to be underestimated since the statistical test is applied to the entire distribution of signal and noise of each subtraction image condition. To overcome some of these problems, a new analysis, developed in our laboratory by Tom Videen (Squire et al., 1991; Videen et al., 1991) was applied to selected PET responses.

Analysis on irregular volumes of interest. This analysis assesses the significance of specific local blood flow changes by defining irregular volumes of interest (VOI) in one group of images, and testing the same regions for a significant change (greater than the noise level) in a second group of images. VOI boundaries were defined on a first group of subjects (see Results) using isocontour boundaries drawn on the mean-difference image. VOI definition began with the maximum pixel for the region; boundaries were then expanded in three dimensions over a set of contiguous pixels while regional $t$ values were computed for each newly defined VOI using the individual component images from the first group. For each region, that VOI which produced the maximum regional $t$ value was used to test the statistical replicability of the response in a second group of subjects. This volume of interest was statistically evaluated in this second group of images using a paired $t$ test with Bonferroni correction for multiple comparisons. This analysis represents a statistically sound strategy to assess formally the regional significance of PET activations. However, its extensive use is somewhat limited by the fact that only a limited number of selected responses can be replicated within onc experiment, since the Bonferroni correction for multiple comparisons tends to lower exceedingly the necessary level of significance for replication.

Since two different statistical analyses were applied to this data set, and only one of them (VOI analysis) accurately estimates the significance level of a particular PET response, we will report as significant only those regions that were replicated with the volume of interest analysis; as activated or active, regions whose $\mathrm{z}$ scores were higher than 1.96 in 
A) SHIFTING ATTENTION TASK $(n=18)$
\[ <0.001 \]

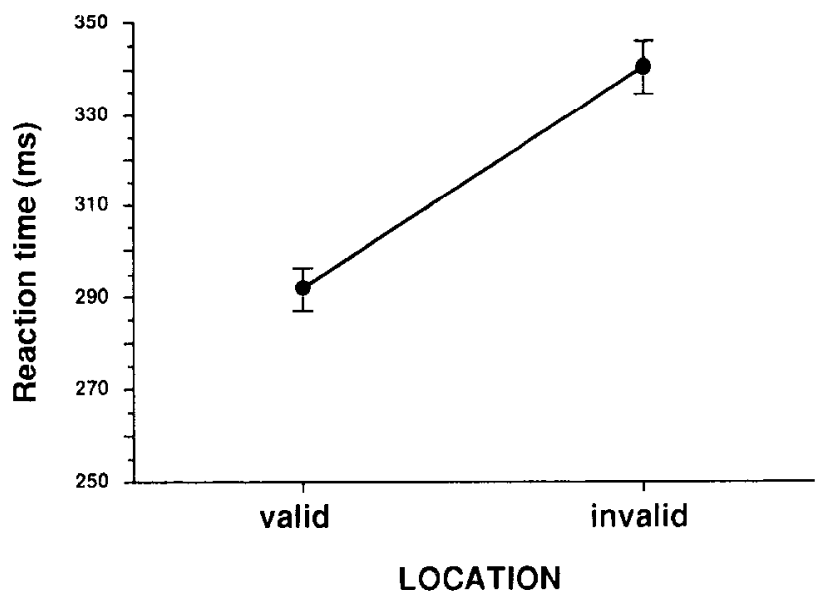

B)

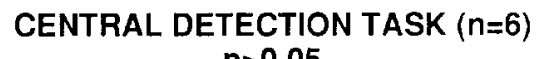
$p>0.05$

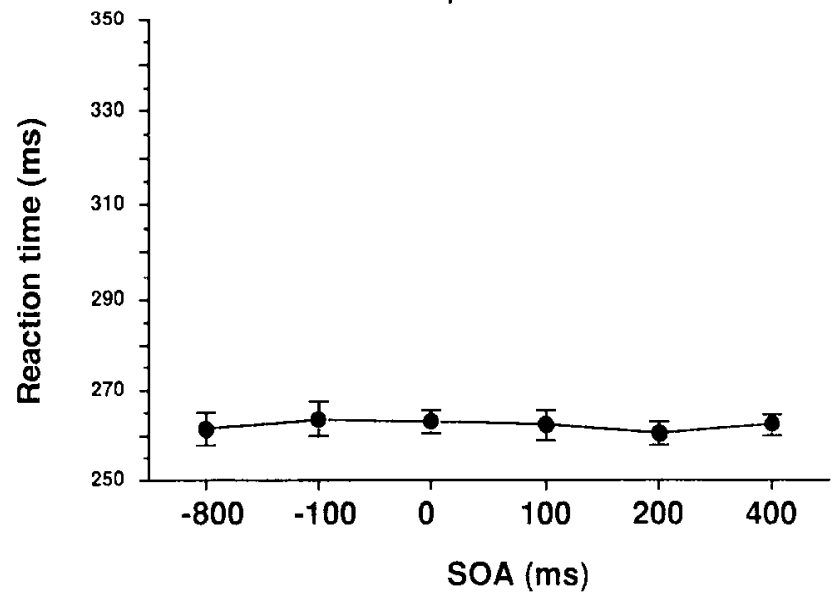

Figure 2. Psychophysical results. A, Shifting attention task, session 1. $B$. Central detection task, pilot study.

the distribution change analysis and were consistently localized in a number of conditions; and as weakly activated or weakly active, regions whose $z$ scores approached 1.96 and were also consistently localized in a number of conditions.

\section{Results}

\section{Psychophysics}

Shifting attention task. The effect of spatial cueing was evaluated by comparing RT for predictable (validly cued) versus unpredictable (invalidly cued) locations. For session 1 (psychophysics), a repeated-measure ANOVA was run on RT with spatial cueing (valid, invalid), visual field (left, right), and direction (left, right) as factors. For session 2 (psychophysics/PET), a repeated-measure ANOVA was run on RT with spatial cueing (valid, invalid), visual field (left, right), direction (left, right), and phase (before injection, during scan, after scan) as factors. Errors (a response with a latency higher than $1000 \mathrm{msec}$ or lower than $100 \mathrm{msec}$, or a missed response) were less than $5 \%$ in both sessions, and were not considered in the analysis.

Peripheral probe stimuli were detected significantly faster at validly than invalidly cued locations [session 1 , mean $\pm \mathrm{SE}$, valid vs. invalid trials: $292 \pm 4.7$ vs. $340 \pm 5.9 \mathrm{msec}, F(1,18)$ $=55.6, p<0.0001$ (Fig. $2 A$ ); session $2,267 \pm 3.0$ vs. $299 \pm$ $4.9 \mathrm{mscc}, F(1,14)=51.1, p<0.0001]$. No consistent differences were found between visual fields [session $1, F(1,18)=0.03, p$ $=\mathrm{NS}$; session $2, F(1,14)=4.38, p=\mathrm{NS}$ ] or direction [session $1, F(1,18)=1.1, p=\mathrm{NS}$; session $2, F(1,14)=1.2, p=\mathrm{NS}]$. No interactions were found among spatial cueing, hemifield, or direction (all interactions in both sessions had an $F$ value less than $2.4, p=\mathrm{NS}$ ). In the PET session, RTs for valid and invalid trials that were collected during the actual blood flow measurement (valid-invalid trial probability $=95 \%-5 \%$ ) were not significantly different from those collected before and after [validinvalid trial probability $=80 \%-20 \% ; F(2,28)=1.02, p=\mathrm{NS}]$. The change in probability for valid and invalid trials did not influence RTs, and went subjectively undetected in all subjects.

In conclusion, subjects responded faster to stimuli presented at valid locations than to stimuli presented at invalid locations. According to models of attention, this performance advantage reflects a processing advantage for stimuli that fall within a region of space previously selected by a covert spatial attention mechanism. Spatial attention was presumably cued by the spatial predictability of the stimuli and the instruction to shift attention voluntarily to the most probable location.

The relative delay for responding to stimuli presented at invalidly cued locations may represent the time needed to redirect attention to the stimulus location after being misdirected toward another location. The spatial distribution of such delays (costs) has been used to investigate the underlying neural representations, that is, neural maps in which attentional operations may be implemented. We compared reaction time on invalid trials to target location-expected location pairings in which the target location occurred in the same or opposite direction of the current sequence of attention shifts. For instance, we compared RTs to an invalid probe at $6^{\circ}$ of visual angle in the right visual field when a $10^{\circ}$ target was the expected location and attention was shifting in a rightward direction (a direction opposite, or away from, the invalid location), to RTs to the same $6^{\circ}$ probe under conditions in which the expected location was again $10^{\circ}$ but attention was shifting in a leftward direction (the same direction, or toward, the invalid location). Since the retinal locations of both the probe and expected location are identical in the two cases, differences in RT must reflect a special cost to changing the direction of attention. This comparison was performed only on trials in which intermediate locations were cued $\left(3^{\circ}, 6^{\circ}\right.$, and $\left.10^{\circ}\right)$ to avoid ambiguities concerning the direction of attention when the sequence of cued locations reset at $1^{\circ}$ and $15^{\circ}$. Data for left and right visual field were independently analyzed. A two-way ANOVA was conducted on RT with visual field (left, right) and momentum (same, opposite) as factors. RTs were significantly longer for invalid trials presented on the opposite side of an ongoing attention movement [same vs opposite, mean $\pm \mathrm{SE}, 319 \pm 8.6$ vs $354 \pm 10, F(1,18)=27.9, p<0.0001]$. Again, no difference between visual fields was evident $[F(1,18)$ $=0.31, p=$ NS $]$.

This difference in RTs between invalid trials on the same or opposite side of an attention movement, however, did not completely account for the difference between valid and invalid trials, that is, the effect of spatial cueing per se. Valid trials were still significantly faster than invalid trials on the same side of an attention movement (keeping target position constant) [mcan $\pm \mathrm{SE}, 295 \pm 6.4$ vs. $319 \pm 8.5, F(1,18)=9.68, p<0.01]$.

When probed and expected locations are one position apart 
Tahle 1. PET subtractions and putative task components

PET subtractions

Shifting attention - fixation point

Passive - fixation point

Central detection - fixation point

Shifting attention - central detection
Putative task components

Peripheral visual stimulation

Peripheral shifts of attention (voluntarily generated)

Planning and execution of motor response to lateralized visual stimulus

Arousal

Peripheral visual stimulation

Peripheral shifts of attention (automatically generated)

Peripheral visual stimulation

Central visual stimulation

Centrally maintained attention

Planning and execution of motor response to central visual stimulus

Arousal

Pcriphcral shifts of attention (voluntarily generated)

Planning of motor response to lateralized visual stimulus (e.g., $6^{\circ}$ and $10^{\circ}$ ), the location counter to the ongoing direction of the attention shift also corresponds to the location of the previously detected probe. This configuration is known to produce a long-lasting inhibition of the response (inhibition of return; Posner and Cohen, 1984), and might explain the momentum effect. We therefore analyzed the momentum effect as a function of the number of intervening positions (one, two, or three) between the cue and the target. Although there was a tendency for a larger momentum effect for cue target pairings that involve fewer intervening positions ( $47 \mathrm{msec}$ for one, 41 msec for two, and $25 \mathrm{msec}$ for three intervening positions), the effect of intervening positions was not significant $[F(2,32)=$ $0.554]$.

In conclusion, there is an additional cost to changing the direction of an attention movement when attention has been misdirected to another location. This momentum effect is distinct from the advantage yielded by correctly cueing a spatial location.

Central detection task. The efficacy of the central detection task in suppressing shifts of attention to peripheral stimuli while detecting foveal events was evaluated in a pilot study. Central probes were presented in the absence or presence of peripheral distractors, which were flashed at different SOAs from the central probes $(-800,-100,0,100,200,400 \mathrm{msec})$. One-way repeated-measure ANOVAs compared RTs to central probes in the presence or absence of peripheral stimuli (collapsing across SOAs), and the effect of SOA. RTs to central probes were slower in the presence than in the absence of peripheral stimulation $[$ mean $\pm \mathrm{SE}, 262 \pm 2$ vs. $247 \pm 5 \mathrm{msec}, F(6,5)=4.1, p<$ 0.011 . In the presence of peripheral distractors, the speed of responding to central events was independent of the SOA between peripheral and central stimuli $[F(5,5)=0.19, p=\mathrm{NS}$; Fig. $2 B$ ], suggesting that attention was not automatically driven to the periphery by the distractors. The global decrement in performance related to the presence of peripheral distractors may represent a shift in response criterion. When distractors are present, subjects cannot respond to any transient but only to those in the center of the field.

In sessions 1 and 2 of the main experiment, peripheral distractors were flashed 100 or $400 \mathrm{msec}$ after the central probes (only central events were relevant for the response). RTs in the central detection task were faster than in the shifting attention task (session 1, central detection vs. shifting attention valid trials, mean $\pm \mathrm{SE}, 242 \pm 5$ vs. $267 \pm 3 \mathrm{msec}$ ). No difference in central RT was found when the peripheral distractors were presented in the left or right field $[F(1,33)=0.05, p=\mathrm{NS}]$.

\section{PET Functional anatomy}

This article considers activations that can be related to shifts of spatial attention in the periphery of the visual field. These activations will reflect only attention-related processes that occur during valid trials, since invalid probes were very rare $(5 \%)$ during the PET scan. The basic criterion is that significant responses should be stronger in the shifting attention than in the central detection task. Distinctions between voluntary and automatic shifts of attention, or the perceptual and intentional aspects of spatial selection, are examined by comparing the shifting attention and passive tasks. We also report responses that, because of their pattern of activation or relationship with the clinical literature, might be related to other visuospatial processes. Finally, we report activations related to the visual stimuli and the execution of a motor response.

Two main image subtractions were used to isolate task-related areas of blood flow change (Table 1). To compare the pattern of activations across conditions, the fixation point only scan was subtracted, as the control scan (-fixation point), from the shifting attention, central detection, and passive scan conditions. To confirm activations that were presumably related to peripheral shifts of spatial attention, we also subtracted each central detection condition (-central detection) from the corresponding shifting attention conditions, thus matching sensory, motor, and arousal factors. The specific processes putatively isolated by the different subtractions are outlined in Table 1.

Because of movement artifacts between scans, the individual subtraction images contributing to each summed image (one for condition) came from partially nonoverlapping groups of subjects, that is, the summed images were not entirely within subjects. The movement problem was particularly evident in this set of data because we compared control (e.g., fixation point) and activation scans (e.g., one of the shifting attention conditions), which were sometimes four scans apart in the series, that is, about $40 \mathrm{~min}$ apart. The overlap among subjects for task subtractions within a hemifield with the fixation point as control [e.g., left visual field shifting attention (left or right direction), central detection, passive] was $75 \%$ (66\% for left field, $84 \%$ right field). The overlap among subjects for conditions across hemi- 


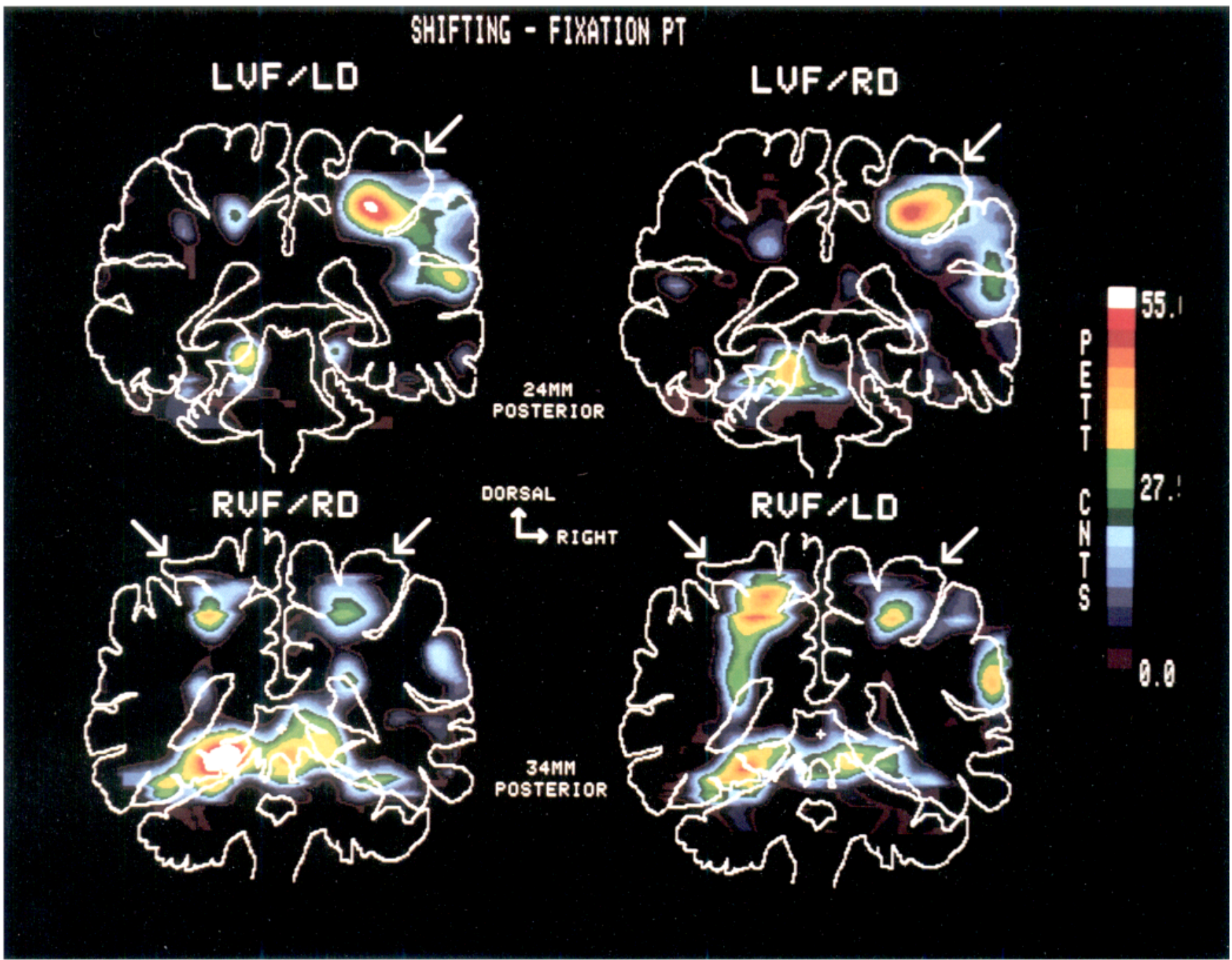

Figure 3. Coronal blood flow PET sections through the posterior parietal cortex for shifting conditions - fixation point control. $L V F / L D$, left visual field/left direction; $L V F / R D$, left visual field/right direction; $R V F / L D$, right visual field/left direction; $R V F / R D$, right visual field/right direction. For each field condition, PET sections are taken through the center of mass of the stronger response. For left field conditions, a weaker activation was localized in the left hemisphere at the same response location as for right visual field conditions. The arrows refer to the location of the superior parietal response.

fields was about $55 \%$. Nevertheless, all subjects contributing to each shifting attention - fixation point image also contributed to the corresponding central detection - fixation point image. The second level subtractions shifting attention - central detection were therefore entirely within the same group of subjects.

Seven of eight images with the fixation point as control were significant to the $\gamma-2$ test at $p<0.01$ level. Only the RPASSFPT image failed to reach significance, but data will be still presented for comparison with the other conditions. Three of four images with the central detection task as control were significant to the $\gamma-2$ test at $p<0.05$ level. Only the LVF/LDLCD image failed to reach significance, but the data will be presented for comparisons with the other conditions.

\section{Regions related to shifts of attention}

Superior parietal cortex. Regions in the superior parietal lobule, around the postcentral sulcus (Talairach et al., 1967; near Brodmann area 7) were activated during the detection of peripheral probes in all four shifting attention conditions when the fixation point control scan was subtracted away. Left visual field detection activated a right superior parietal region, near the postcen- tral sulcus, and a left superior parietal region more posteriorly (Figs. 3, 4; Table 2). The magnitude of the contralateral response was twice as large as the magnitude of the ipsilateral response. Left and right direction conditions activated the same regions at the same magnitude level. Right visual field detection conditions activated a left superior parietal region, near the one activated by left visual field detection, and a distinct right superior parietal region, posterior to the focus for left detection (Figs. 3, 4; Table 2). These right field responses seemed more bilateral, although still stronger in the contralateral parietal cortex for the left direction condition. Left and right direction conditions again activated similar cortical foci. Figure 4 summarizes the location (center of mass) and magnitude of the foci in the superior parietal lobe that were activated by the various shifting attention tasks. While in the left superior parietal cortex the foci for left and right visual field detection clustered in the same region (for both direction conditions), in the right superior parietal cortex the foci for left visual field detection were consistently localized more anteriorly and laterally than the foci for right visual field detection (for both direction conditions). The average vector distance between these two regions of activation 


\section{Shifts of visuo-spatial attention}

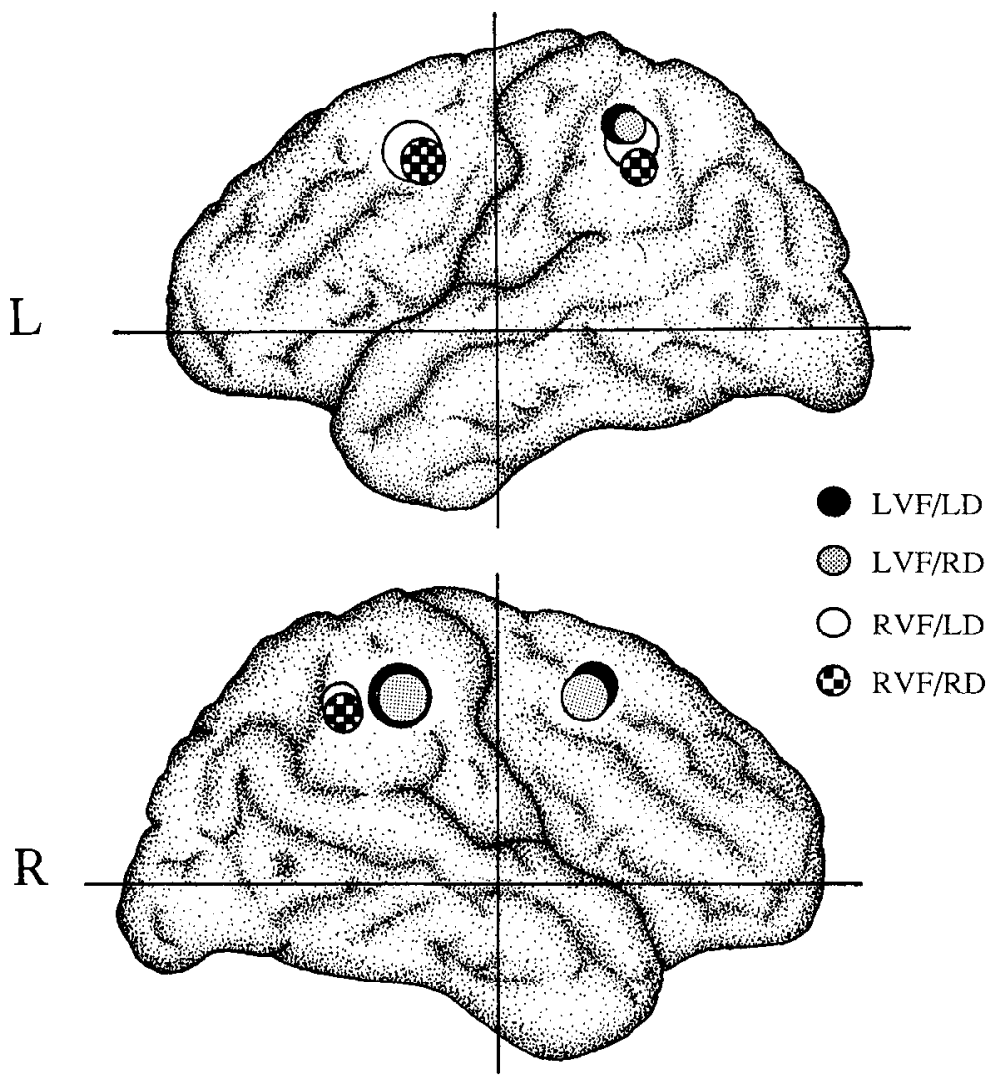

Figure 4. Location of superior parietal and frontal responses in shifting attention conditions. Abbreviations are as in Figure 3. The area of each symbol is proportional to the magnitude in PET counts at the center of mass of the response. The control subtraction conditions are fixation point for the parietal, and central detection for the frontal activations.

in the right hemisphere was about $18 \mathrm{~mm}$. This vector distance and its consistency across conditions in a mostly overlapping set of subjects suggest the presence of a single representation in the left superior parietal lobe, and a separate representation for each field in the right superior parietal lobule.
To assess formally the reproducibility of these superior parietal responses, the boundaries of each region of activation (contralateral and ipsilateral) were defined, independently for each visual field, in one direction condition (left direction for left field, and right direction for right field), and their repro-

Table 2. Foci of activations in superior parietal cortex

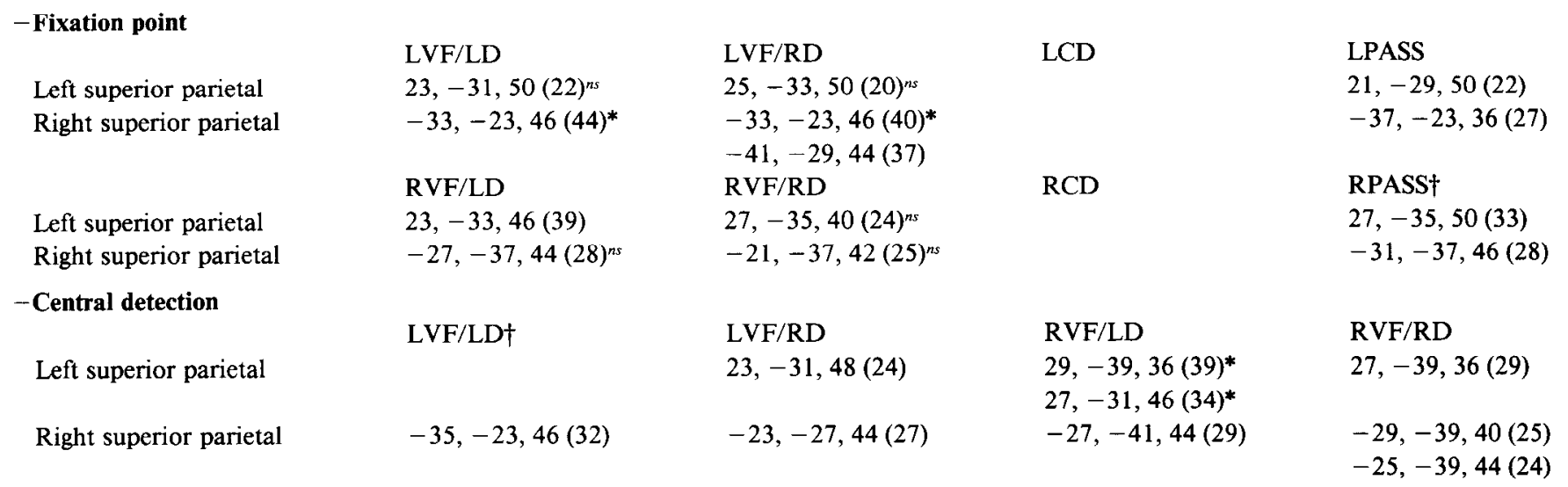

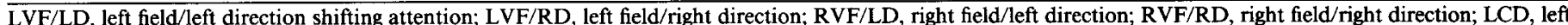

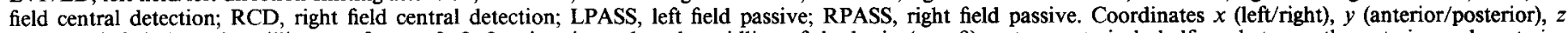

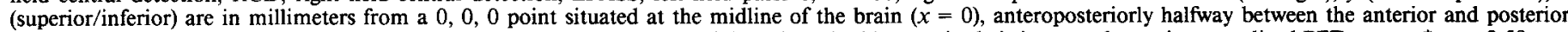

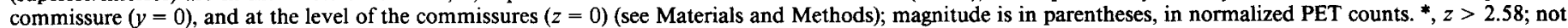
marked, $z>1.96 ; n s, z<1.96 ; \dagger$, not significant image according to distribution change analysis (see Materials and Methods). 
Table 3. Replication analysis on superior parietal and frontal foci

\begin{tabular}{|c|c|c|c|c|c|c|}
\hline Region of interest & $\begin{array}{l}\text { Hypothesis- } \\
\text { generating group } \\
(n)\end{array}$ & Magnitude $^{a}$ & $\begin{array}{l}t \text { (de- } \\
\text { fining) }\end{array}$ & $\begin{array}{l}\text { Replication group } \\
(n)\end{array}$ & Magnitude $^{a}$ & $t$ (replication) ${ }^{c}$ \\
\hline \multicolumn{7}{|l|}{ Left visual field } \\
\hline Contralateral superior parietal & Left direction (13) & $49 \pm 7.6$ & 6.43 & Right direction (15) & $34 \pm 10.1$ & $3.3 p<0.005$ \\
\hline Ipsilateral superior parietal & Left direction (13) & $32 \pm 5.4$ & 5.89 & Right direction (15) & $16 \pm 7.6$ & $2.1 p<0.06$ \\
\hline Contralateral superior frontal & Left direction (10) & $31 \pm 3.9$ & 3.67 & Right direction (12) & $36 \pm 7.0$ & $5.0 p<0.0005$ \\
\hline \multicolumn{7}{|l|}{ Right visual field } \\
\hline Contralateral superior parietal & Right direction (16) & $38.6 \pm 13.1$ & 2.94 & Left direction (12) & $44 \pm 13.5$ & $3.2 p<0.005$ \\
\hline Ipsilateral superior parietal & Right direction (16) & $32.3 \pm 7.1$ & 4.54 & Left direction (12) & $27 \pm 8.5$ & $3.2 p<0.005$ \\
\hline Contralateral superior frontal & Right direction (11) & $46 \pm 11.0$ & 4.01 & Left direction (13) & $30 \pm 10.0$ & $3.01 p<0.01$ \\
\hline
\end{tabular}

${ }^{a}$ Magnitude is given as mean \pm SE in normalized PET counts within the region of interests, respectively in the hypothesis and replication group.

${ }^{b} t$ (defining) is the optimized $t$ value for the indicated region based on the subjects contributing to the hypothesis-generating group (one direction condition).

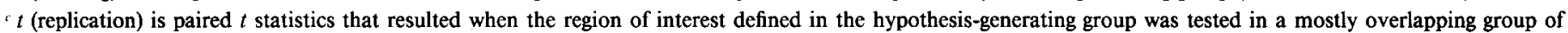

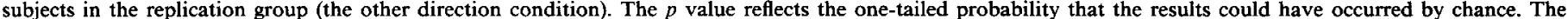

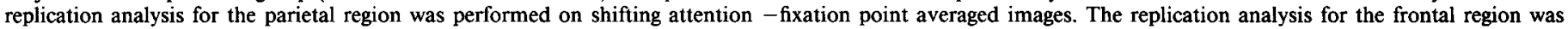
performed on shifting attention - central detection averaged images.

ducibility was tested in the other direction condition (right direction for left field, and left direction for right field). Since two regions were evaluated for each visual field, the significance level was adjustcd to $p=0.025$ with a Bonferroni correction $(p / N$ where $p=0.05$ and $N=2$ ). As indicated in Table 3, the contralateral and ipsilateral activations for right visual field attention, and the contralateral activation for left visual field attention were significantly replicated. The ipsilateral activation for shifting attention in left visual field did not achieve significance. In conclusion, shifting attention to the left visual field mostly activated right superior parietal cortex, whereas shifting attention to the right visual field significantly activated both left and right superior parietal cortex.

There was no detectable superior parietal activation during the central detection task (Fig. 5, Table 2), where subjects were responding to foveal probes and presumably not attending to the peripheral stimuli. In contrast, responses were localized at similar locations in the passive tasks, where subjects were presumably automatically attending to the peripheral stimuli (Fig. 5 , Table 2). The response magnitude in the contralateral hemisphere for left field passive was lower than for left field shifting conditions, while it was comparable in both hemispheres between right field passive and shifting conditions. Again left and right field passive conditions activated a similar region in the left superior parietal lobule, but spatially distinct regions in the right superior parietal lobule (see Table 2).
When each central detection task was subtracted from the corresponding shifting attention tasks, thereby matching visual, motor, and arousal factors, similar superior parietal activations were still localized (Table 2).

In conclusion, these results generally support a relationship between shifts of attention (internally cued or automatic) to the peripheral visual field and activity in regions of the superior parietal cortex. This superior parietal activity appears to encode the visual field of an attention shift rather than its direction within a field. Attention to the left visual field yields mostly right superior parietal activity, while attention to the right visual field yields more bilateral activations (left and right superior parietal). Attention to the left and right visual field also seems to activate a similar region in left superior parietal cortex, but distinct regions in right superior parietal cortex.

Superior frontal cortex. Superior frontal cortex (near superior area 6 of Brodmann) was consistently activated by all four shifting attention tasks. Because of the proximity with motor and supplementary motor cortex, these regions were best imaged when the motor activity related to the speeded left hand key response (see below) was subtracted away by using the central detection task as control condition (Table 4). These regions were also contralateral to the field in which attention was directed, and were similarly activated by either direction (Fig. 6).

To assess formally the reproducibility of the superior frontal response, its boundaries were defined in one direction condition

\section{Table 4. Foci of activations in superior frontal cortex}

\section{-Fixation point}

$$
\text { Left superior frontal }
$$

Left superior frontal

- Central detection

Left superior frontal

Right superior frontal

\section{LVF/LD}

RVF/LD

$21,17,42(46)^{*}$

LVF/LD $\dagger$

$-31,23,48(31)$
$\mathrm{LVF} / \mathrm{RD}$

$23,11,48(44)^{*}$

RVF/RD

$17,12,50(39)^{*}$

LVF/RD

$-35,21,46(32)^{*}$
LCD

$\mathrm{RCD}$

RVF/LD

$23,19,44(40)^{*}$
LPASS

RPASS $\dagger$

$19,5,52(28)$

Details are as in Table 2 . 


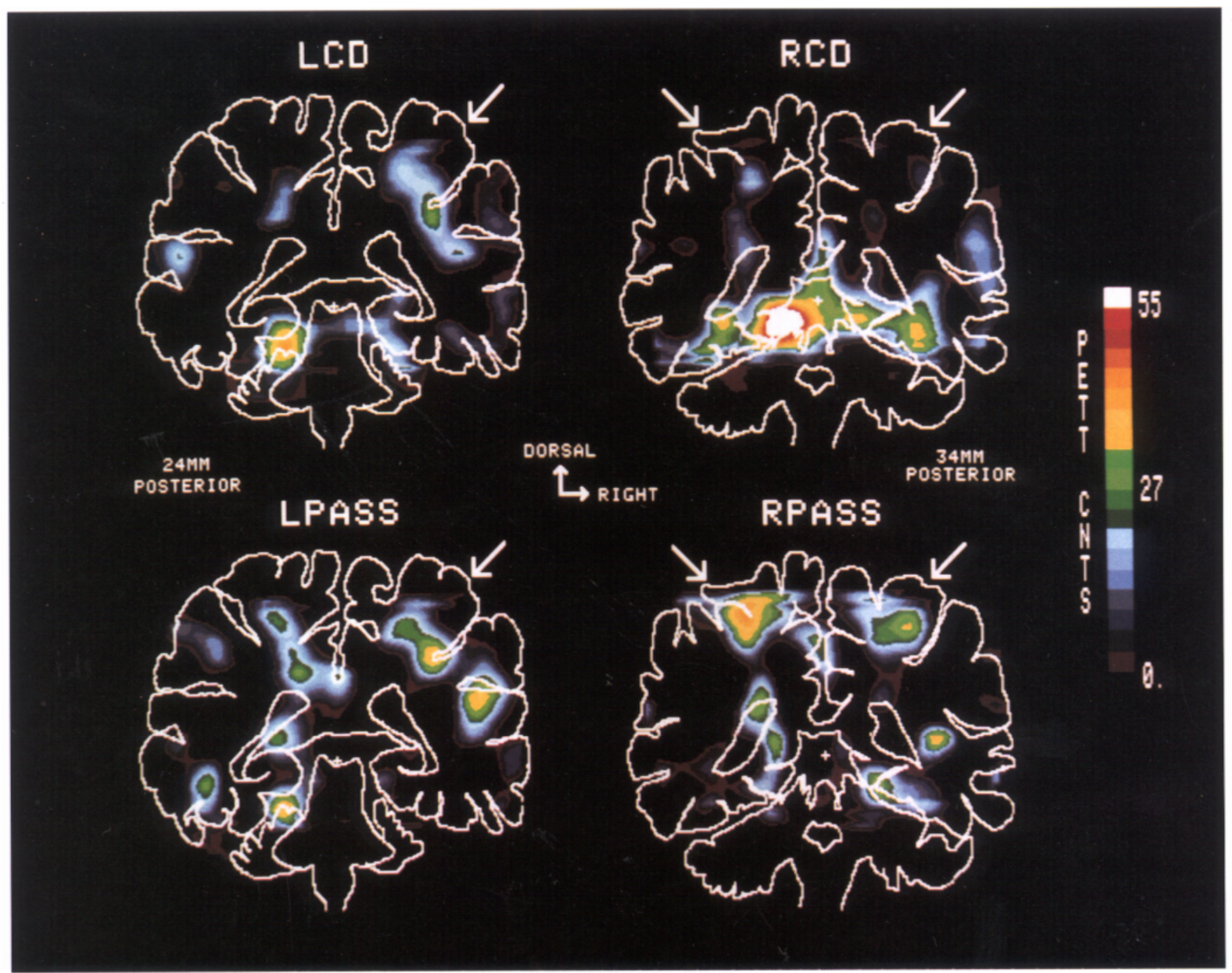

Figure 5. Coronal blood flow PET sections through the posterior parietal cortex for central detection and passive conditions - fixation point control. $L C D$, central detection, left visual field distractors; $R C D$, central detection, right visual field distractors; $L P A S S$, left visual field passive; $R P A S S$, right visual field passive. The arrows refer to the location of the superior parietal response in Figure 3.

(left direction for left field, and right direction for right field), and its reproducibility was tested in the other direction condition (right direction for left field, and left direction for right). As indicated in Table 3, the contralateral superior frontal response was replicated in both field conditions.

Since the superior parietal region was driven by internally cued (shifting attention task) as well as automatic shifts of attention (passive tasks), it is of interest to examine the superior frontal region in the passive task. Figure 7 shows a sagittal section through the superior frontal region in the left hemisphere during right visual field shifting attention, central detection, and passive tasks with the fixation point condition as control scan. Significant responses are evident in the shifting attention tasks (similarly for the two directions), but not in the central detection and passive tasks (Table 4). A similar comparison was not possible during left visual field condition because, as mentioned, motor related activations masked this response in the right frontal cortex. In conclusion, a contralateral superior frontal region was activated when attention was directed into the contralateral visual field and stimuli were overtly detected by a key press response. The same region was not active when the same pe- ripheral stimuli were presented and attention was maintained in the center, or when attention was presumably directed to the peripheral stimuli but without overt detection.

\section{Other visuospatial processing regions}

Inferior parietal cortex/superior temporal cortex. The clinical literature clearly points to the right inferior parietal lobule, and right parietotemporal junction as the site of damage most frequently associated with symptoms of hemineglect. We localized responses in the inferior parietal lobule (supramarginal gyrus, near Brodmann area 40), and superior temporal cortex (superior temporal sulcus, superior and middle temporal gyrus, near Brodmann area 22) only in some conditions. The inferior parietal region was mostly activated for left field shifting attention and passive conditions. The superior temporal cortex was mostly activated for right field shifting attention, passive, and central detection conditions (Table 5). Some of these responses were above and some below the $1.96 \mathrm{z}$ score cutoff in the distribution change analysis. All activations were right lateralized in agreement with the clinical literature. No response was evident at these locations when the central detection was subtracted from 


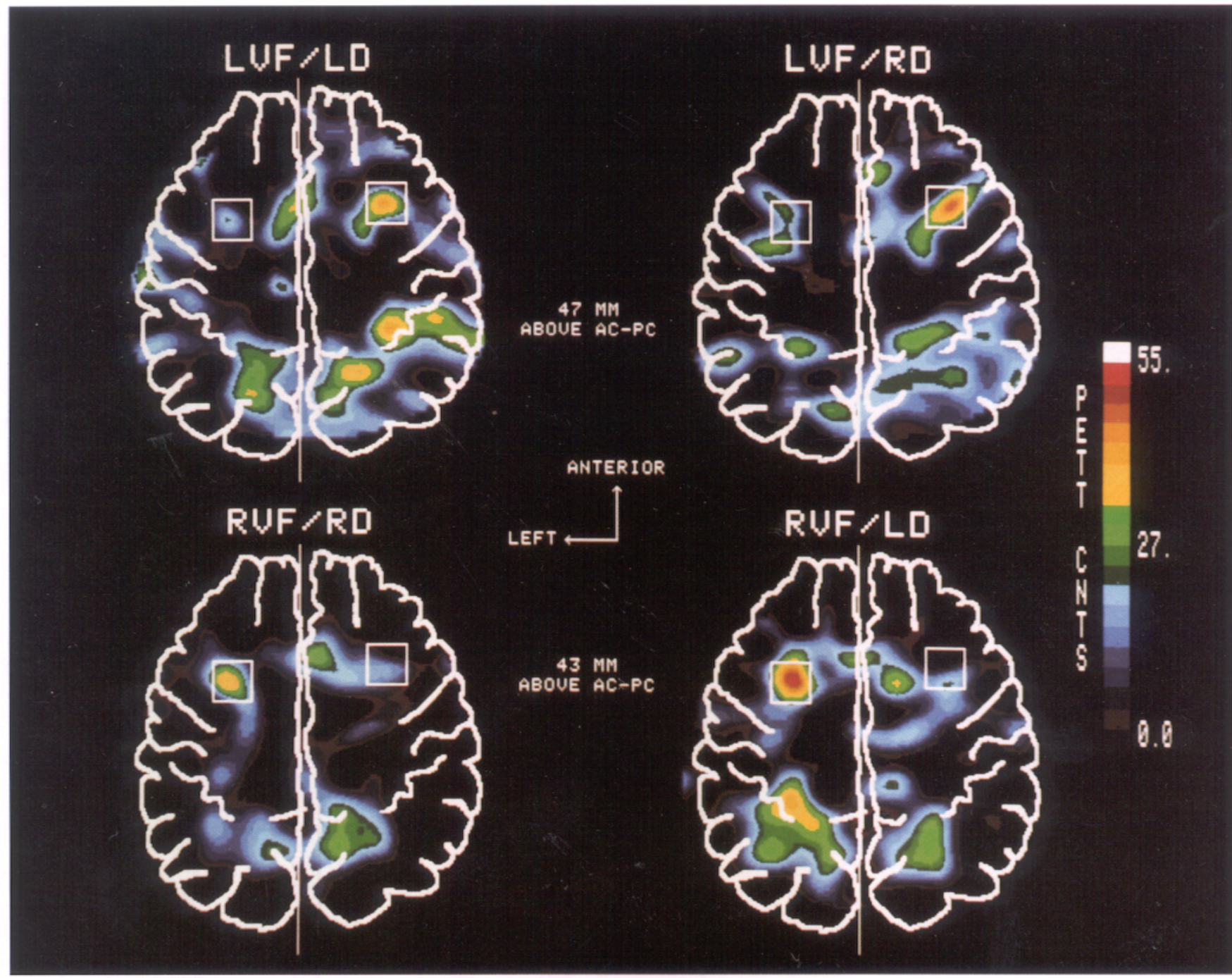

Figure 6. Horizontal blood flow PET sections through superior frontal cortex for shifting attention - central detection conditions. Abbreviations are as in Figure 3.

the shifting attention condition. In summary, right inferior parietal and superior temporal cortex were inconsistently activated by this particular set of tasks. Nonetheless, the right hemisphere dominance was consistent with the clinical literature, suggesting that this region may contribute to the cortical control of attention.

Precuneus and other parietal foci. Regions in medial parietal cortex (precuneus, near medial area 7) were activated only for left visual field attention when the fixation point control was subtracted away, but were bilaterally active in all conditions when the central detection condition was substracted away (Table 5). This suggests that these regions were similarly active during the shifting attention and fixation point condition, and relatively hypoperfused during central detection condition. Finally, other lateral superior parietal and posterior cingulate regions were active in some of the left visual field conditions (Table 5).

Anterior cingulate cortex. A region in the anterior cingulate (near Brodmann area 24) was active in all shifting attention conditions after subtracting away the central detection condition. Given the proximity with supplementary motor area (SMA), which was strongly activated in all conditions with a key press response (see below), it is not clear whether the anterior cingulate response represents true activity from the cingulate cortex or a relative difference in SMA activation between shifting attention and central detection task. This region was not active when the fixation point was subtracted from the passive task. However, a region located about $14 \mathrm{~mm}$ more posteriorly in the middle portion of the cingulate gyrus was significantly active in both passive conditions (Table 5).

\section{Visual regions}

Lingual/fusiform regions were activated in most task conditions when the fixation point control was subtracted away. This subtraction matched the visual activity related to the static array of boxes, which was also displayed when subjects looked at the fixation point, and isolated activity related to the brief transients that were flashed at different locations. Foci were strictly contralateral for the peripheral stimuli (in the shifting attention, central detection, and passive tasks), and bilateral for the foveal stimuli (in the central detection task). The location was quite variable (Table 6), presumably because the dim sensory stimulus did not strongly drive these visual regions. Furthermore, for right visual field shifting attention and central detection tasks, 


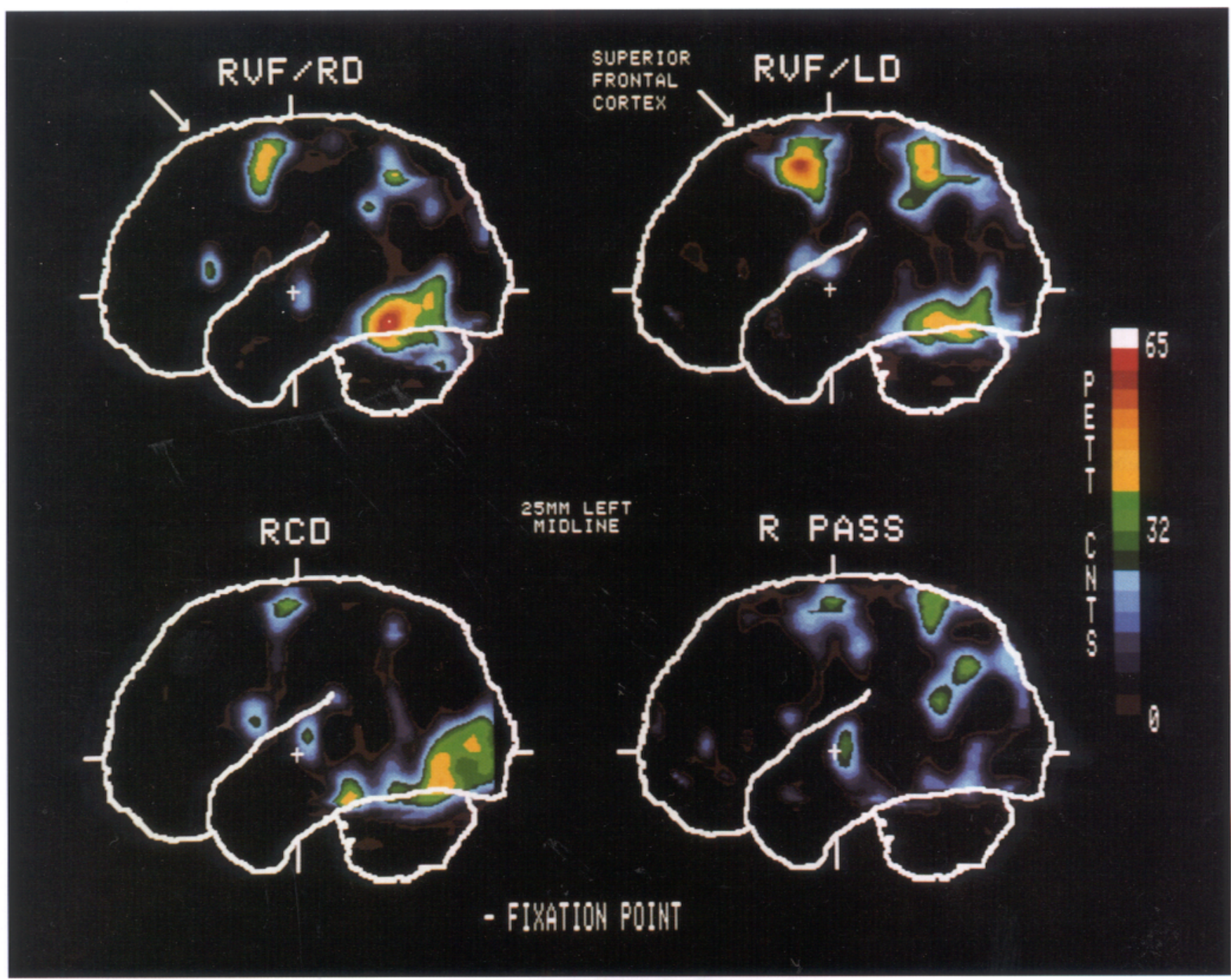

Figure 7. Sagittal blood flow PET section through left superior frontal cortex for right visual field shifting attention, central detection, and passive conditions - fixation point control. Abbreviations are as in Figures 3 and 5.

activity in left ventral occipital regions overlapped with activity coming from the superoanterior portion of the cerebellum strongly activated by the motor response (see below). Activity in these regions returned to the noise level in the second level subtraction, shifting attention-central detection, suggesting that the visual response to the peripheral stimuli was not significantly enhanced by the selection of the stimulus location.

\section{Motor regions}

Primary sensory-motor cortex and supplementary motor cortex. Contralateral primary sensorimotor cortex, and bilateral (stronger contralaterally) SMA were activated (against a fixation point baseline) when subjects responded to the visual stimuli (shifting attention, central detection) with the left hand (Fig. 8). Interestingly, the magnitude and extent of both motor cortex and SMA response were greater when the stimuli were presented in the visual field ipsilateral to the responding hand, independently of direction (Table 7, Fig. 9). The same effect was also present in the central detection task, where the relevant stimuli were foveal. Stronger SMA and motor cortex responses were obtained when ipsilateral rather than contralateral distractors were pre- sented (Table 7, Fig. 9). Motor cortex magnitude was 68\%, 91\%, and $71 \%$ larger for left than right visual field stimuli, respectively, in shifting attention, left and right direction, and central detection task. SMA magnitude was $43 \%, 39 \%$, and $18 \%$ larger for left than right visual field stimuli, respectively, in shifting attention, left and right direction, and central detection tasks. This pattern of activations suggests a possible dependence of motor/premotor activity upon the correspondence between the visual field of incoming stimuli and the hand used in responding.

Cerebellum. The superoanterior portion of the cerebellum was also activated by the key press response. The activation was strictly ipsilateral to the responding hand (left) when subjects responded to lateralized left visual field stimuli (left shifting attention), and was more bilateral when subjects responded to foveal stimuli (left or right central detection) or lateralized right visual stimuli (right shifting attention; Table 7, Fig. 10). Although field-by-hand magnitude effects were not observed in the cerebellum as in motor and supplementary motor cortex, the pattern of activation again suggests some dependence of activity upon sensory information.

Inferior frontal cortex. A region of inferior frontal cortex (near inferior Brodmann area 6) was activated during shifting atten- 
Table 5. Foci of activations in inferior parietal/superior temporal cortex, precuneus, other parietal foci, anterior cingulate cortex

Inferior parietal cortex/temporal cortex

Fixation point

Right supramarginal

Right superior temporal/middle temporal

Right supramarginal

Right superior temporal/middle temporal

Precuneus

-Fixation point

Right precuneus

\section{-Central detection}

Right precuneus

Left precuneus

Other parietal foci

\section{-Fixation point}

Left posterior cingulate

Right lateral superior parietal

Left lateral superior parietal

Right lateral superior parietal

\section{-Central detection}

Right lateral superior parietal

Left lateral superior parietal

Anterior cingulate cortex

\section{Fixation point}

Right middle cingulate

Left middle cingulate

\section{- Central detection}

Right anterior cingulate

Left anterior cingulate

$$
\begin{aligned}
& \text { LVF/LD } \\
& -61,-25,20(29)^{\mathrm{ns}} \\
& \text { RVF/LD } \\
& -63,-37,20(27)^{\mathrm{ns}} \\
& -63,-17,8(25)^{\mathrm{ns}}
\end{aligned}
$$

LVF/LD

$-17,-35,40(30)^{\mathrm{ns}}$

LVF/RD

$-13,-35,42(27)^{\mathrm{ns}}$

LVF/LD $\dagger$

$-15,-43,50(32)$

$-19,-41,48(31)$

$-11,-53,34(30)$

$19,-37,50(32)$

$11,-35,50(30)$

LVF/LD

$19,-15,36(34)$

$-59,-39,38(32)$

RVF/LD

RVF/RD

$$
\begin{aligned}
& \mathrm{LVF} / \mathrm{LD} \dagger \\
& -55,-21,50(30) \\
& -52,-25,34(30)
\end{aligned}
$$

LVF/RD

$-58,-40,37(24)$

$-35,-39,42$ (24)

$-48,-23,-51(23)$

$29,-39,30(31)^{*}$

$23,-31,48$ (24)

LVF/LD

RVF/LD

LVF/LD $\dagger$

$-3,29,36(48)$
LVF/RD

RVF/RD

LVF/RD

$-5,35,38(27)$

$-7,34,44(26)$
LCD

PASS

$-55,-23,22(28)$

$\mathrm{RCD}$

$-69,-15,-4(29)$

$-69,-15,0(28)$

LCD

LPASS

$-15,-33,34(33)^{*}$

$-13,-41,42(29)^{*}$

RVF/LD

$-19,-43,50(28)$

$-13,-29,42(23)$

$19,-35,44(32)^{*}$

$9,-45,48$ (27)

LCD

RCD

RVF/RD

$-15,-41,48(30)^{*}$

$9,-45,42(25)$

LPASS

$17,-17,36(34)^{*}$

$3,-11,30(31)^{*}$

$1,-29,30(25)$

$-55,-37,32(23)$

$-35,-51,36(23)$

RPASS $\dagger$

$27,-47,30(27)$

$-35,-49,32(26)$

RVF/LD

RVF/RD
LCD

RCD

RVF/LD

$-15,17,42(26)$

$-5,29,38(24)$

$9,21,32(26)$
LPASS

$-7,5,34(35)^{*}$ $-9,19,34(35)^{*}$ RPASS $\dagger$

$11,15,30(32)$

$13,15,40(29)$

RVF/RD

$-7,27,42$ (24) 


\begin{tabular}{|c|c|c|c|c|}
\hline -Fixation point & & & & \\
\hline & LVF/LD & LVF/RD & LCD & LPASS \\
\hline Right primary visual & & & & $\begin{array}{l}-13,-69,12(26) \\
-5,-67,-6(24)\end{array}$ \\
\hline Right lingual/fusiform & $-27,-43,0(41)$ & & $\begin{array}{l}-33,-59,0(43)^{*} \\
-41,-41,-8(37)^{*} \\
-33,-43,0(35)^{*} \\
-35,-37,2(34)^{*}\end{array}$ & $-17,-33,-8(37)^{*}$ \\
\hline Left lingual/fusiform & & & $\begin{array}{l}23,-29,-14(37)^{*} \\
27,-43,-12(29) \\
42,-50,-14(28) \\
19,-49,-2(27)\end{array}$ & $-43,-51,-18(29)^{*}$ \\
\hline Left hippocampus & & & & $21,-19,-16(29)^{*}$ \\
\hline Right middle temporal & RVF/LD & $\mathrm{RVF} / \mathrm{RD}$ & $\mathrm{RCD}$ & $\begin{array}{l}-45,-31,4(26) \\
\text { RPASS }\end{array}$ \\
\hline Left lingual/fusiform & $31,-29,-14(45)^{*}$ & & $\begin{array}{l}29,-59,-6(35)^{*} \\
27,-61,-2(34)^{*} \\
37,-93,-8(30) \\
37,-47,-2(28)\end{array}$ & $\begin{array}{l}39,-57,-4(29)^{*} \\
37,-53,10(27)\end{array}$ \\
\hline Right lingual/fusiform & & & $\begin{array}{l}-27,-45,-2(35)^{*} \\
-29,-51,6(33) \\
-39,-39,-4(33) \\
-21,-27,-12(27)\end{array}$ & \\
\hline Left lateral occipital & & & & $\begin{array}{l}39,-53,18(28) \\
37,-55,4(28)\end{array}$ \\
\hline Left hippocampus & & & $\begin{array}{l}23,-17,-16(31) \\
19,-19,-14(30)\end{array}$ & $15,-21,6(28)$ \\
\hline Right middle temporal & & & $\begin{array}{l}-43,-41,0(34)^{*} \\
-43,-49,16(27)\end{array}$ & $-45,-45,4(26)$ \\
\hline
\end{tabular}

Contralateral foci (e.g., right visual cortex for LVF conditions) are listed first. Other details are as in Table 2.

tion, passive, and central detection tasks (Table 7, Fig. 8). Activations were contralateral to the responding hand when a key press response was executed, and bilateral in the passive condition. This region can be considered neither strictly motor related, since some activity was recorded in the passive task in which no overt motor response was performed, nor strictly shifting attention related, since some activity was recorded in the central detection task.

\section{Discussion}

Luminance detection tasks involving a spatial manipulation of target probability (spatial cueing) were used in a group of normal volunteers to localize regions of the human brain that were active when attention was shifted in the left or right visual field, along a left or right direction. In a first session, the efficacy of these tasks in controlling attention was verified psychophysically. In a second session, the related functional anatomy was mapped with PET activation methodology. These main results were obtained:

(1) Two cortical regions, one in superior parietal and one in superior frontal cortex, were more active when attention was shifted across various locations than when maintained at the center of gaze.

(2) Both superior parietal and frontal activations encoded the visual field and not the direction of an attention shift. Furthermore, in the right hemisphere parietal responses for selection of the left and right visual field were spatially segregated.

(3) Superior parietal and superior frontal cortex were related to different aspects of spatial selection. The parietal region was active when peripheral locations were selected on the basis of cognitive or sensory cues independent of the execution of an overt response. The frontal region was active only when responses were made to stimuli at selected peripheral locations.

(4) Activation of several motor-related regions was influenced by the relationship between the visual field of stimulus presentation and the side of the responding hand. Motor and supplementary motor cortex were more strongly active for stimuli presented ipsilaterally to the responding left hand.

\section{Psychophysical effects}

The psychophysical data support the hypothesis that the shifting attention task induced shifts of attention across visual locations while the central detection task locked attention in the center of gaze.

In the shifting attention task, where a lateralized sequence of targets was presented with a mostly foveofugal or foveocentric direction, stimuli at correctly cued locations (valid trials) were responded to faster than stimuli at incorrectly cued locations (invalid trials). The difference in RT between valid and invalid targets suggests that subjects used foreknowledge of target location to select the cued location.

These shifts of attention were probably driven by cognitive rather than reflexive processes. Although attention can be directed by a symbolic cue, such as an arrow pointing toward the most likely target location, attention can also be drawn reflexively by a transient (Jonides, 1981; Muller and Rabbitt, 1989). 


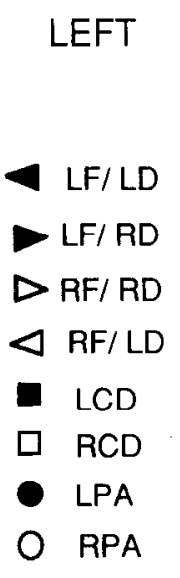

$\mathrm{R} \mid \mathrm{GHT}$
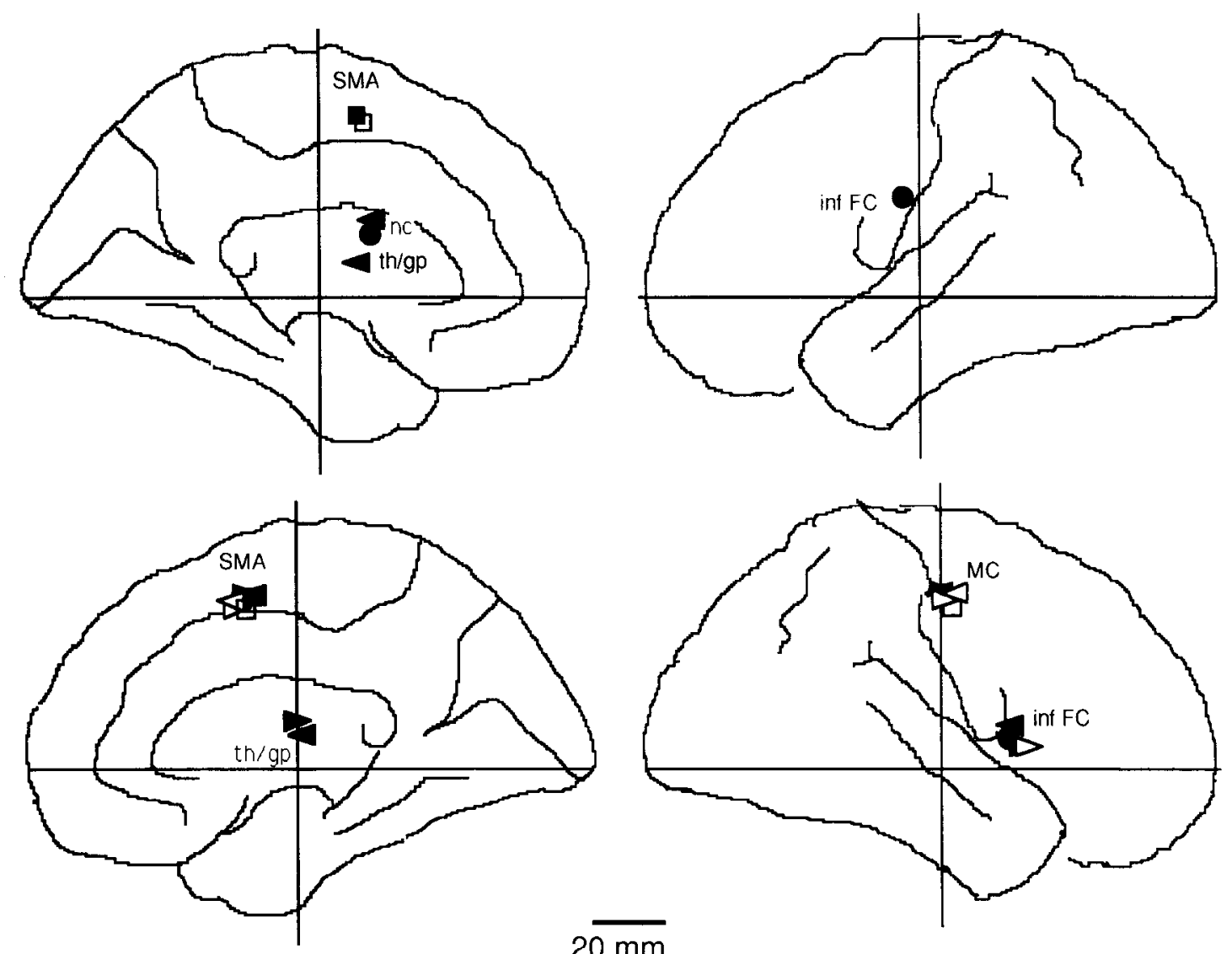

$20 \mathrm{~mm}$

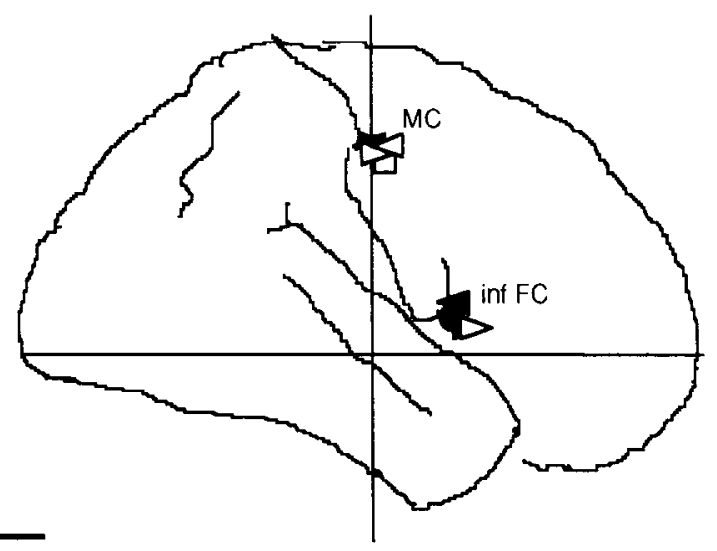

Figure 8. Outline of blood flow activations in motor-related regions. Shifting attention, central detection, passive condition - fixation point control. inf $F C$, inferior frontal cortex; $M C$, motor cortex; $n c$, nucleus caudatus; $t h / g p$, thalamus/globus pallidus. $L F, R F$, left, right visual field; $L P A, R P A$, left, right visual field passive.

In the present experiment, the appearance of the target would reflexively draw attention to that location. However, this transient could not direct attention reflexively to the upcoming target location, while the highly significant pattern of costs and benefits suggests that such a shift did occur. Furthermore, reflexive shifts of attention produce a facilitation for time intervals much shorter than the 1500-2000 msec intertarget interval used in the present study.

Predictable probes (valid trials) were responded to equally fast in either visual field and direction, but the direction of attention influenced the speed of detection of unpredictable probes (invalid trials). RTs to unpredictable probes were faster when they were presented in the same, rather than in the opposite, direction of an ongoing attention movement. For example, when a $10^{\circ}$ location was expected, RT to a $3^{\circ}$ invalid target was faster when the sequence of preceding attentional shifts was toward the fovea than when away from the fovea. Control analyses indicated that this effect probably did not reflect inhibition of return. When probed and expected locations are one position apart (e.g., $6^{\circ}$ and $10^{\circ}$ ), the location counter to the ongoing direction of the attention shift also corresponds to the location of the previously detected probe. This configuration is known to produce a long-lasting inhibition of the response (inhibition of return; Posner and Cohen, 1984), but the direction effect in the present study did not significantly change with the spatial interval separating the cue and target locations.
The effect of the direction of attention on invalid trials is perhaps better explained by the notion that recomputing the direction and amplitude (or retinal distance) of an attention shift takes longer than recomputing the amplitude (or retinal distance) only. This result is consistent with other studies demonstrating that RT cost is increased when the cue and invalid target are presented in opposite fields with respect to the vertical or horizontal meridians (Hughes and Zimba, 1987; Rizzolatti et al., 1987; Tassinari et al., 1987). In both cases, shifting attention from the cue to the target requires a change in direction (left and right, up and down). It is important to note that only the facilitatory effect of valid spatial cueing, not the cost associated with invalid cueing, was imaged by the corresponding blood flow measurements since invalid trials were rare during the scan.

In the central detection task, targets at the center of gaze were responded to equally quickly when lateralized peripheral distractors were presented before, simultaneously, or after the central stimuli. The independence of RTs to central targets from the SOA of the peripheral distractors suggests that attention was mostly engaged at the center of gaze and rarely shifted toward the peripheral distractors. Yantis and Jonides (1990) have recently shown that by focusing attention on a location, it is possible to suppress the automatic tendency to orient toward the abrupt onset of luminance transients.

No psychophysical data were collected during the passive 


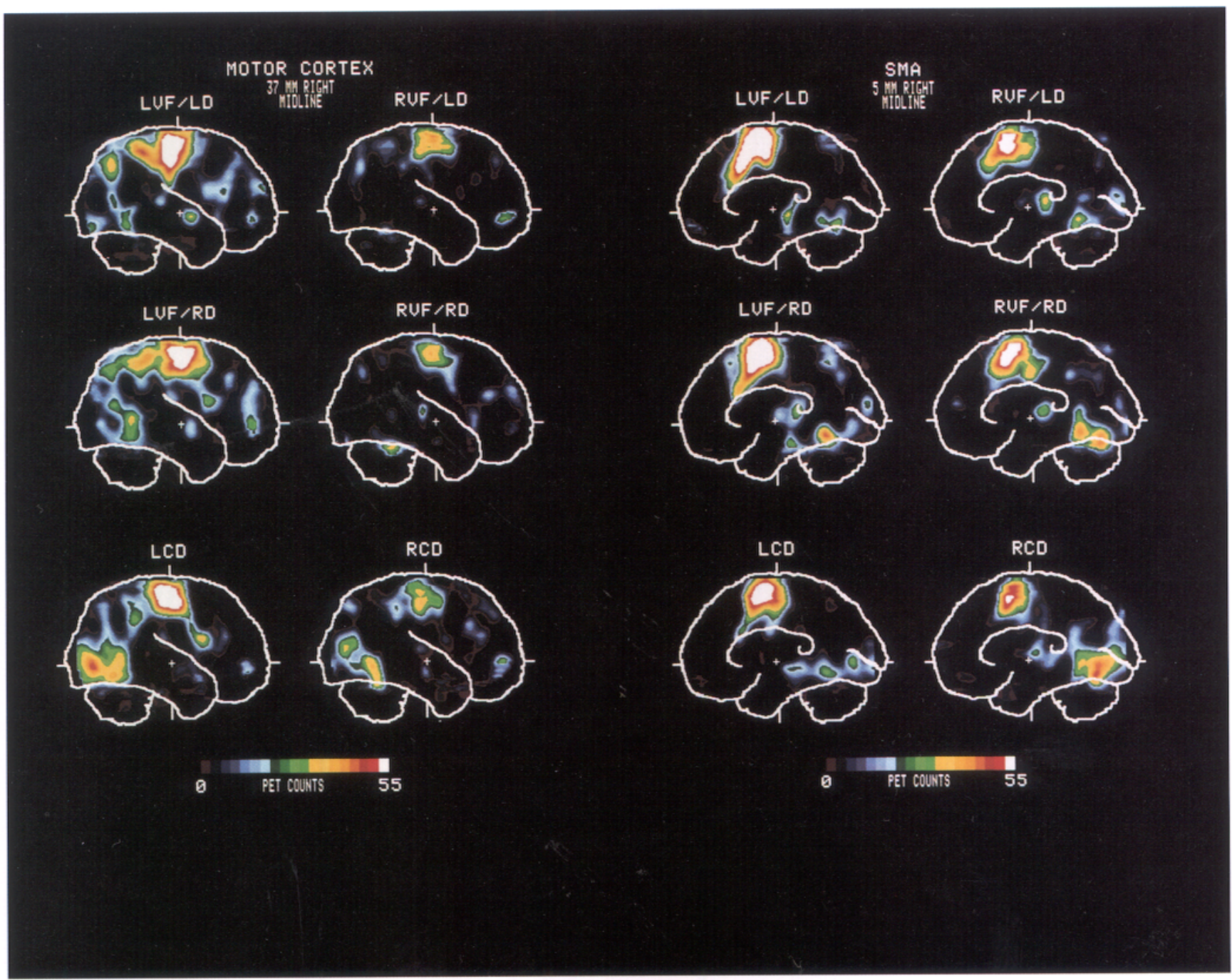

Figure 9. Sagittal blood flow PET sections through primary motor cortex (left) and supplementary motor cortex (right) for shifting attention and central detection condition - fixation point control. Abbreviations are as in Figures 3 and 5.

task, where stimuli were lateralized to one or the other field (as in the shifting attention condition) but were not overtly detected. Although the subject's behavior was not explicitly monitored, it is likely that attention was automatically shifted toward the peripheral stimuli. On each trial before the stimulus presentation, attention was presumably not engaged on any particular task or location. The degree of central focusing was certainly lower than in the central detection task, in which foveal stimuli were actively detected. Peripherally, each of the five possible locations of stimulation was equiprobable, precluding any possibility of spatial expectation. Such a state of "diffuse" attention (Eriksen and Yeh, 1985) is known to be very sensitive to the onset of abrupt peripheral transients (Jonides, 1981; Muller and Rabbitt, 1989; Yantis and Jonides, 1990). Reflexive shifts of spatial attention during the passive task therefore probably occurred, enabling comparisons of PET activations produced by shifts of attention driven reflexively or by cognitive processes.

\section{PET Functional anatomy}

Regions related to shifts of spatial attention

A region was considered to be related to shifts of spatial attention when it was more active in the shifting attention and passive task, than in the central detection task.
Superior parietal cortex. A region in superior parietal cortex (near area 7 of Brodmann) was consistently more active during shifting attention and passive conditions than during central detection conditions, in comparison to a fixation point only baseline. This region remained significantly active when the central detection condition was directly subtracted from the shifting attention condition, matching motor, most sensory, and arousal factors.

Residual sensory differences between shifting attention and central detection tasks cannot plausibly account for this response. The pattern of peripheral visual stimulation was predictable in the shifting attention task, but random in the central detection task, while foveal transients were only presented in the latter condition. These differences seem irrelevant to the observed differences in the parietal responses. Furthermore, these regions were also active during the passive task, in which the pattern of peripheral stimulation was random as in the central detection task, and no foveal stimuli were presented as in the shifting attention task.

Another possibility is that the superior parietal activation in the shifting attention and passive conditions represents a simple peripheral visual response, which is gated or filtered out when attention is foveally focused during the central detection task. 
Table 7. Foci of activations in motor-related regions

\section{-Fixation point}

Right sensory-motor cortex

Supplementary motor cortex

Left cerebellum

Right cerebellum

Right inferior frontal

Left inferior frontal

Right thalamus/globus pallidus

Left thalamus/globus pallidus

Left caudatus

Right primary sensorimotor cortex Supplementary motor cortex

Left cerebellum

Right cerebellum

Right inferior frontal

$\begin{array}{ll}\text { LVF/LD } & \text { LVF/RD } \\ -37,-1,50(62)^{*} & -35,1,50(63)^{*} \\ -5,13,50(87)^{*} & -7,15,50(78)^{*} \\ 15,-35,-12(41) & 7,-39,-12(62)^{*} \\ 19,-51,-16(40) & 23,-49,-16(46)^{*} \\ -65,19,12(43) & -55,23,6(34) \\ -21,-1,-1(51)^{*} & -17,0,14(35) \\ & -15,-3,10(34)\end{array}$

\section{LCD}

$-37,0,50(60)^{*}$

$-5,11,50(59)^{*}$

$5,11,52(56)^{*}$

$19,-27,-14(38)^{*}$

$23,-29,-14(37)^{*}$

$27,-43,-12$ (29)

$-17,-34,-10(40)^{*}$

LPASS

$-65,19,8(26)$

$57,5,29(26)$

$13,11,10(38)$

$9,15,22(32)$

RVF/LD

$-37,3,50(37)$

$0,19,48(61)^{*}$

\section{RVF/RD}

$-37,1,48(33)$

$-5,17,46(56)^{*}$

$13,-27,-8(36)$

$15,-23,-14(35)$

$9,-48,-10(44)^{*}$

$21,-49,-14(40)^{*}$

$-21,-45,-12(39)$

$19,-31,-12(56)^{*}$

$1,-48,-12(50)^{*}$

$-21,-44,-12(36)$

$-11,-35,-8(31)$

\section{RCD}

$-43,3,46(35)^{*}$

$7,13,50(49)^{*}$

$-1,15,46(48)^{*}$

$13,-31,-9(57)^{*}$

$25,-51,-10(39)^{*}$

$21,-43,-14(34)$

$-3,-47,-8(43)^{*}$

$-17,-45,-12(42)^{*}$

$-10,-47,-12(41)^{*}$

$-59,25,6(38)$

\section{SUPERO-ANTERIOR CEREBELLUM}

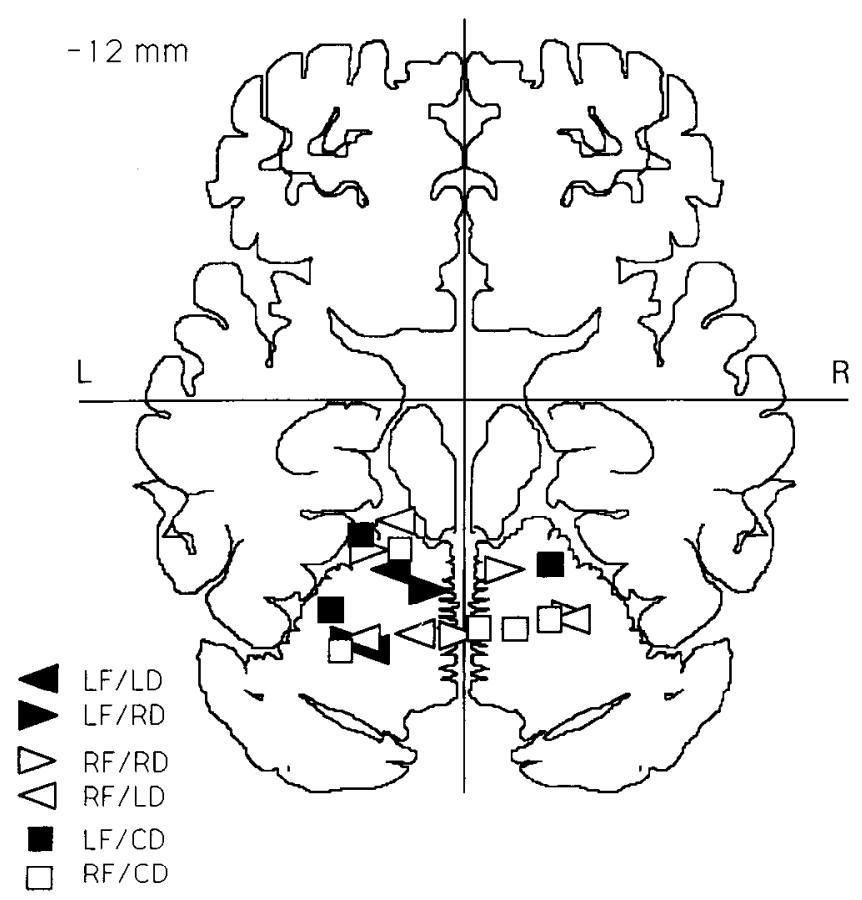

Figure 10. Outline of blood flow activations in cerebellum (superoanterior). Shifting attention, central detection - fixation point control. $C D$, central direction. Other abbreviations are as in Figure 8.
A similar effect has been reported in monkey's inferior temporal cortex (Richmond et al., 1983; Sato, 1989). There are several arguments against this possibility. In one experiment (Petersen et al., in press), attention was tonically directed toward or away from a peripheral stimulus. When these two conditions were subtracted, occipital-parietal but not superior parietal activation was found. If the superior parietal response in the present report was due to a sensory response that is gated when the stimulus is unattended, and is not related to shifts of attention, then superior parietal activation should have been found in Petersen et al. (in press). Also, the superior parietal activations in passive and shifting attention conditions were asymmetrically distributed in the two hemispheres. There was a single focus in the left hemisphere, and separate foci in the right hemisphere for stimulus presentation in the left and right visual fields. The human lesion literature supports the notion of consistent functional asymmetries in a mechanism involved in shifts of spatial attention rather than sensory processing (e.g., Posner et al., 1984; Morrow and Ratcliff, 1988).

A more likely interpretation for the superior parietal response is that it is related to shifts of visual attention across target locations. Shifts of attention would likely occur during both shifting attention and passive conditions, but not during the central detection condition. This interpretation is supported by our psychophysical findings and the cognitive literature on automatic orienting of attention for abrupt transients (Muller and Rabbitt, 1989; Yantis and Jonides, 1990). The similarity in response location for shifting attention and passive conditions can be explained by assuming that the same shifting mechanism was recruited on the basis of cognitive or sensory cues. 


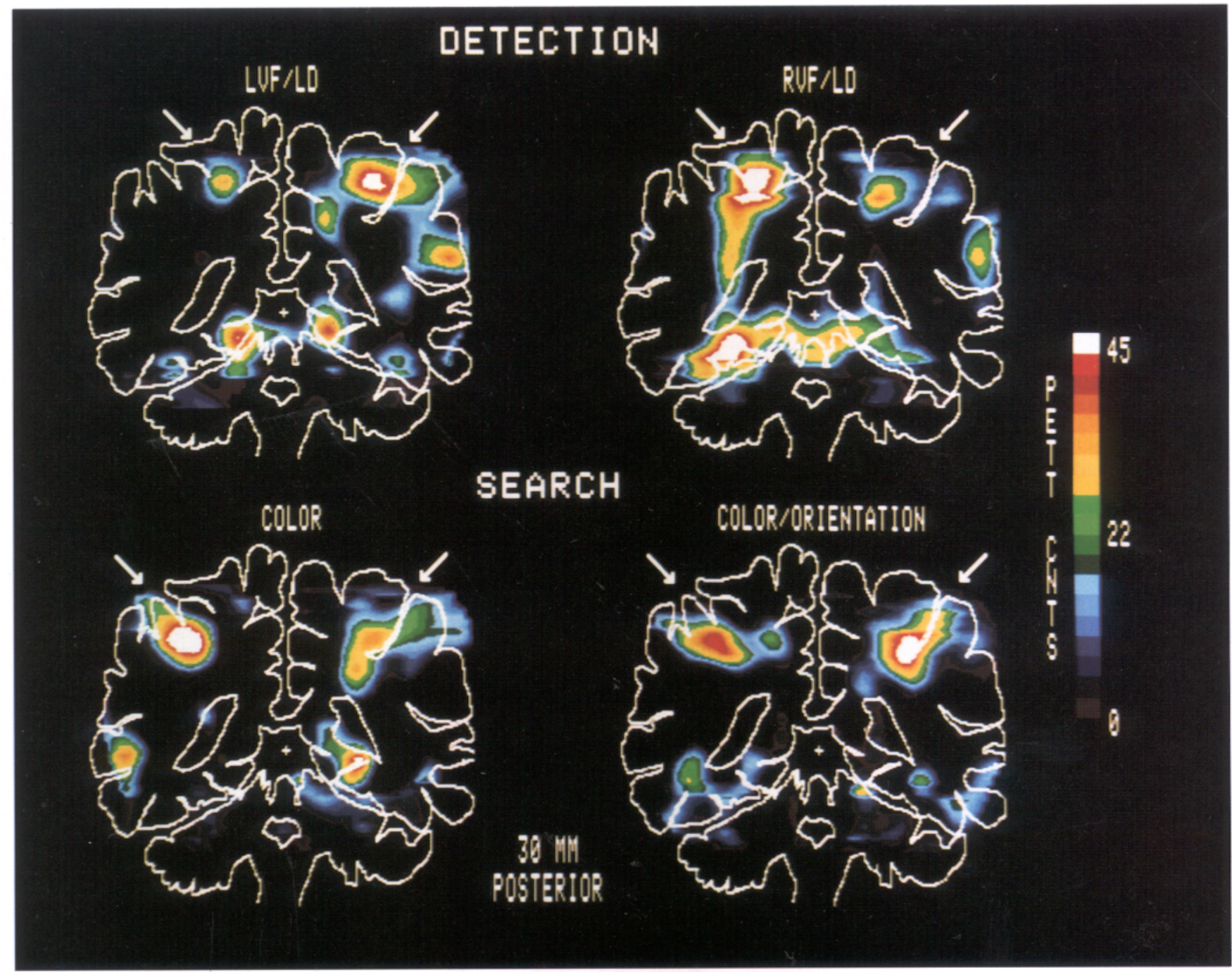

Figure 11. Coronal blood flow PET sections through posterior parietal cortex during luminance detection (present experiment) and visual search task (Corbetta et al., 1991b). Detection, Shifting attention - fixation point control. Search, Covert recognition of a color (color) or conjunction (color/orientation) target in an array of four similar objects (see Discussion) - passive viewing of the same set of stimuli. Abbreviations are as in Figure 3. The arrows refer to the location of superior parietal activity.

There are differences, however, between the shifting and passive conditions. In the passive task, attention was briefly drawn to the periphery by the onset of the stimulus and then presumably returned to the center, while in the shifting task attention, was engaged for a longer time at the upcoming probe location. The strong effect of the ongoing direction of the attention shift on reaction time to invalid probes suggests that a return of attention to the center was suppressed during the shifting task. Finally, a motor response was associated with a shift of attention only in the shifting task. It is unclear if superior parietal activation was equivalent in the passive and shifting conditions. The magnitude of the contralateral superior parietal response was greater for three of four shifting conditions relative to the corresponding passive condition and equivalent for all four ipsilateral responses. These subtle differences, if real, between shifting and passive conditions might be explained by any of the factors listed above.

Superior parietal activation has been detected during other tasks involving shifts of spatial attention across object locations. In a visual search paradigm (Corbetta et al., 1991b; M. Corbetta, unpublished observations), subjects looked for a target defined by a single feature (e.g., color) or a conjunction of features (e.g., color and orientation). The targets were individual geometrical objects (e.g., a red square or a red bar) that could appear randomly at any of four different locations. Significant PET responses were localized in inferior temporal cortex, probably related to target recognition, and in superior parietal cortex. Figure 11 shows the responses in the superior lobule obtained during the visual search task for feature and conjunction targets, and the ones obtained in the present detection task. Despite differences in stimulus eccentricities, visual features, task demands (recognition of a simple object vs. the detection of a luminance change), and the underlying brain anatomy (different group of subjects in the two experiments), the similarity in response location with the present study is good (the vector distance between the two regions of activation was about $16 \mathrm{~mm}$ ). This region appears to be recruited when attention is shifted toward locations or object locations, independent of the current task demands or goals.

Superior parietal cortex was not active in other tasks that demanded attention to visual stimuli without shifts of spatial attention. No superior parietal activation was found, as previ- 
ously mentioned, when attention was tonically maintained on a lateralized peripheral visual stimulus (Petersen et al., in press). Nor was superior parietal activation seen when attention was directed to the color, motion, or speed of a multi-object display during a match-to-sample discrimination (Corbetta et al., 1990, 1991a). In the latter task, the activity of a different set of extrastriate visual regions was modulated for each task-relevant feature, and these regions appeared to be specialized for processing the relevant feature. The nature of the display and task, which did not require shifts of spatial attention from one object to another, suggests that these effects reflected mechanisms related to feature selection rather than spatial selection.

In summary, the activation of a region in superior parietal cortex (as monitored through maps of blood flow with PET) may correlate with shifts of visual attention across locations or object locations. This region can be similarly recruited through cognitive or sensory cues, and across a variety of perceptual tasks (luminance detection, present report; feature and conjunction target recognition, Corbetta et al., 1991 b; imagery, Roland and Friberg, 1985) encouraging shifts of attention. The pattern of activation is thus location dependent and task independent, resembling a "shifter" mechanism able to shift the window of processing to different field locations (Ullman, 1984). Shifts of spatial attention are needed whenever information from a selected location must be read out or processed, independently of how and/or why that particular location has been selected.

The same region is not active when attention is directed to other stimulus features (motion, color, shape). Selecting a location, therefore, activates a set of brain regions (at least one other region in frontal cortex was selectively active in this experiment; see below) that are distinct and dissociable from those activated by selecting a stimulus feature. These results argue for the functional independence of the two selection mechanisms. This is consistent with ERP findings that directing attention to the location or features of a target stimulus produces different temporal electrocortical modulations (Harter et al., 1982; Hillyard and Munte, 1984).

One or more computations could be implemented in the superior parietal cortex and be related to shifts of spatial attention. Cognitive analyses have suggested that the focus of processing is shifted from one to another location (or object) through a series of simpler operations (Posner et al., 1984). Attention must "disengage" from the current object of interest, "move" to a new object, and "engage" it. In a cluttered environment, a different or complementary mechanism may be needed for filtering out irrelevant distractors (LaBerge and Brown, 1989). This model is supported by lesion work in humans and monkeys, which indicates that damage to different brain regions selectively impairs these different operations (Posner et al., 1984, 1987a; Robinson et al., 1985; Rafal and Posncr, 1987; see Colby, 1991, for a review of animal studies). Posterior parietal lesions in particular seem to affect the ability to disengage attention from stimuli in the ipsilesional side, when attention must be shifted contralesionally (Posner et al., 1984; Baynes et al., 1986; Morrow and Ratcliffe, 1987). At the single-unit level, Robinson et al. (1991) have recently isolated a population of neurons in posterior parietal cortex that fires as long as attention is directed away from a target location, during spatial detection paradigms similar to the one used in humans. This signal is analogous to a "disengage" operation as defined in Posner's terms. This disengage operation should be operative during both valid and invalid trials, but may be more strongly activated during the latter. Since very few invalid trials were presented during the scanning phase of the present study, blood flow activations may not strongly reflect the disengage component.

Finally, a shift of attention involves the computation of the spatial coordinates of the next intended location, that is, location encoding. Superior parietal neurons may compute these coordinates. Single-unit studies have indicated that neurons in posterior parietal cortex may encode a visual location by integrating retinotopically organized visual signals with actual or intended eye position signals (Andersen et al., 1985, 1990b; Duhamel et al., 1992). ${ }^{1}$

The superior parietal response seems to be controlled more by visual field than direction information. Attention to the left or right visual field predominantly activated the contralateral superior parietal cortex. The direction manipulation had no effect on the magnitude or location of the blood flow response for the left field condition, and only a marginal effect on the magnitude for the right field condition. Although left and right directions in the right field conditions activated similar response locations, the magnitude of the response was stronger in the left hemisphere for leftward than for rightward direction.

The absence of any clear direction effect on parietal cortex activation might be artifactual, reflecting a failure of the behavioral paradigm to induce consistent shifts of attention in a specified direction. The foveofugal or foveocentric series of probes (and, we assume, of shifts of attention) were designed to avoid movements of attention back to the fixation point, which would have inevitably confounded the direction manipulation. The strong effect of the ongoing direction of the attention shift on reaction time to invalid probes, however, suggests that the direction of attention was controlled in the intended manner.

These results do not support a theory of spatial attention based on hemispheric directional biases (Kinsbourne, 1977, 1987). According to this theory, each parietal lobe controls shifts of attention in a contraversive direction, independently of the visual field of stimulus presentation. Moreover, the left hemisphere generates a relatively stronger "contraversive" bias than the right hemisphere, and the two hemispheres act in a dynamic "push-pull" fashion to equilibrate these directional biases. Left hemisphere lesions would lessen this asymmetry, while right hemisphere lesions would worsen it. Unilateral neglect represents an exaggerated tendency to orient rightward, generated by a hyperfunctioning and unopposed left hemisphere, and this mechanism could explain the much higher incidence of left over right unilateral neglect. Two straightforward predictions can be derived from the directional hypothesis in the present experiment: (1) stimuli changing location with a rightward direction within the left or right visual field should mostly drive the left parietal lobe, and vice versa; (2) stimuli changing location with a rightward direction should produce greater parietal activity than stimuli changing location with a lcftward direction. Both

\footnotetext{
${ }^{1}$ No significant activation was detected in the pulvinar (or thalamus) in any of the tasks of the present experiment. The pulvinar is considered part of the circuitry controlling spatial attention in primates, and it may act as a mechanism for increasing the salience of a selected object in a cluttered environment (LaBerge and Brown, 1989). It is not clear how this proposal may fit our negative PET findings. In the shifting attention task, no irrelevant targets were filtered out and one would not expect pulvinar activation. On the other hand, in the central detection task, peripheral distractors were presumably filtered out, yet no thalamic activation was observed. It is possible that more extreme "filtering" manipulations are needed to activate the pulvinar with PET. There is a report in the literature of pulvinar activation during recognition and discrimination tasks in presence of irrelevant distractors (LaBerge and Buchsbaum, 1990). We have preliminary evidence for pulvinar activation during the detection of dim luminance changes in presence of very salient distractor stimuli (S. E. Petersen and M. Corbetta, unpublished observation).
} 
predictions were not confirmed. Moving attention contraversively or ipsiversively in either visual field generally activated similar parietal regions (in terms of both location and magnitude). The only counter example, that is, a relatively stronger left superior parietal lobe response for leftward versus rightward direction, was against Kinsbourne's directional hypothesis.

The presence of a field effect on PET activity suggests that the underlying processing component, that is, shifts of spatial attention in our interpretation, may be organized in visual field coordinates. Similar field-dependent modulations of activity have been reported with PET and ERP recording techniques during other spatial attention paradigms. Petersen et al. (in press) have reported that when attention is tonically maintained on a lateralized dimming target, visual-related activity for a nearby neutral probe stimulus (a slice of a counterphase-flickered checkerboard annulus) is enhanced in a contralateral occipitoparietal region (near upper lateral area 19/39). Several ERP studies (Harter et al., 1982; Mangun et al., 1987) have reported a contralateral amplitude enhancement of early occipitoparietal ERP components when attention was directed to a lateralized location. Although different cortical regions are activated in PET, respectively, by directing attention to a single location or shifting it across locations, these findings would suggest that similar fieldorganized representations are employed.

The parietal cortex was asymmetrically activated by attending to the left or right visual field. Each visual field condition activated the same region in the left superior parietal cortex (more strongly for right field attention), but two distinct regions in the right superior parietal cortex. One interpretation for these findings is that the superior parietal cortex controls lateralized shifts of attention in visual field coordinates, but asymmetrically for the two hemifields. Attention to the left field is mostly controlled by one region in right parietal cortex, while attention to the right field is controlled more bilaterally by a left parietal and a distinct right parietal region. The right superior parietal cortex may then contain the neural hardware to direct attention contralaterally and ipsilaterally.

A potential confound for this interpretation is that all subjects used their left hand to respond to stimuli in either visual field. The parietal asymmetry could be related to a visuomotor interaction between hand of response and visual field of stimulus presentation. The processing was intrahemispheric for left visual field attention (right visual cortex/right motor cortex), and interhemispheric (left visual cortex/right motor cortex) for right visual field attention. Transfer of information through the callosum presumably occurred only in the latter condition, and might relate to the higher bilaterality of the parietal activation during right visual field attention. Recent findings in split brain patients have suggested that the integrity of the posterior portion of the corpus callosum, connecting left and right occipital and parietal lobes, may be critical for such transfer during simple visuomotor tasks (S. Aglioti, G. Tassinari, and G. Berlucchi, personal communication). There are other results, however, that would argue against this possibility. During the passive task, which did not require any motor response and obvious intraor interhemispheric visuomotor transfer, a similar pattern of parietal asymmetry was observed on the response location. Furthermore, there is evidence that all three superior parietal regions (two right and one left) were activated during a visual search task in which bilateral shifts of attention were performed to recognize feature or conjunction targets, which were presented randomly in left or right visual field (M. Corbetta and S. E. Petersen, unpublished observation).
The superior parietal region localized in this PET experiment may correspond to one or more areas of the monkey parietal lobe. In monkey, the superior parietal lobe is mostly somatosensory (Brodmann area 5), and the inferior parietal lobe is mostly visual (areas 7a, LIP, DP, MST) (Ungerleider and Desimone, 1986; Andersen, 1989; Andersen et al., 1990a; Colby and Duhamel, 1991). Area $7 \mathrm{~b}$ in the inferior parietal lobule is predominantly driven by somatosensory stimuli (Hyvarinen, 1981; Andersen, 1989). The homologies between areas in the posterior parietal cortex in monkeys and humans are unclear. According to Brodmann (1909), the human inferior parictal lobule is formed by cytoarchitectonic fields 39 and 40 , phylogenetically newer and presumably related to language function. The superior parietal lobule (fields 5 and 7) would then include both somatosensory and visual areas localized in monkey's parietal lobe. In contrast, according to Von Bonin and Bailey (1947) the cytoarchitectonic organization of the parietal lobe would be similar in the two species (Eidelberg and Galaburda, 1984). The evidence from PET mapping studies in normal volunteers so far suggests that (1) different regions in the superior parietal lobule are activated during spatial processing (Haxby et al., 1991), spatial selection (present report), visual vigilance (Pardo et al., 1991), and cued arm movements (Deiber et al., 1991); (2) different regions in the inferior parietal lobule have been activated by attention to the speed of a visual array (Corbetta et al., 1990), and by repetitive and cued arm movements (Colebatch et al., 1991; Deiber et al., 1991); and (3) language-related tasks tend to activate the posterior superior temporal gyrus more than the inferior parietal lobule (Raichle et al., 1991; Wise et al., 1991). It is our opinion that both superior and inferior parietal lobules may mediate visuospatial processing, while language-related regions are localized more ventrally and rostrally in the superior temporal gyrus.

Superior fronlal corlex. A region of superior frontal cortex (near superior area 6) was active when attention was directed to the contralateral visual field and the visual stimuli were detected with a key press response (shifting attention task). Activations were similar for either direction of attention. The superior frontal region was not active when attention was maintained at the center of gaze and central stimuli were detected (central detection task), or when attention was directed peripherally and stimuli were not overtly detected (passive task).

This frontal activation may reflect several different processcs. The absence of activation in the passive condition suggests that the activity is response related or related to the use of attended information. Yet it does not simply occur whenever a response was made to an attended stimulus, since no activity was recorded in the central detection condition. The central detection condition differs from the shifting attention conditions in that attention is maintained on a single object rather than shifted between objects, and a response is made to a central stimulus rather than to a peripheral stimulus. The frontal activation reported here does not occur, therefore, unless a response is coupled with a shift of attention or a response is made to a lateralized visual stimulus. The subject's response, a key press, was not lateralized. Nonetheless, it is natural to respond to a lateralized visual stimulus with a directed response, and the activation might reflect the preparation of such a response in a region of area 6 . Area 6 is visuomotor and premotor in nature (Wise, 1987; Passingham, 1988). Damage to frontal cortex produces in both humans (Heilman and Valenstein, 1972; DeRenzi, 1982) and monkeys (Kennard, 1939; Latto and Cowey, 1971; Rizzolatti et al., 1983; Deuel and Collins, 1984) unilateral neglect 
of the contralateral space. In humans, frontal damage seems to affect particularly the exploratory-motor components of directed spatial behavior (Bisiach et al., 1990; Daffner et al., 1990). This is consistent with the proposal that perceptual and intentional components of spatial attention are mediated within posterior parietal and frontal cortical areas, respectively (Mesulam, 1981, 1990). It should be noticed, however, that peripersonal and extrapersonal spatial behaviors are differentially affected in monkey by lesions placed respectively in inferior area 6 or frontal eye field (Rizzolatti et al., 1983). Different frontal PET responses might be obtained by directing attention to peripersonal space.

The frontal activation may also reflect an endogenous selection component that was activated only when attention was shifted on the basis of cognitive cues. Theories of prefrontal cortex suggest that this part of the brain may be important for "representational behavior" (Goldman-Rakic, 1987, 1988), that is, behavior guided or planned on the basis of information stored within the nervous system. Recently, Frith et al. (1991) and Deiber et al. (1991) have compared PET activations during conditions of response selection based on internal or external cues, and reported more prefrontal activations in the former condition.

A final possibility is that this activation is eye-movement related. To maintain fixation, subjects may need to suppress eye movements in the active condition, but not in the passive or central conditions since the former required responses to peripheral stimuli. For the same reason, subjects may have prepared and/or executed some small eye movements only in the shifting conditions. Experiments on visually-guided eye movements have localized a putative frontal eye field region (Fox et al., 1985b; M. Corbetta, F. M. Miezin, M. E. Raichle, and S. E. Petersen, unpublished observations) situated between precentral and second frontal gyrus just in front of primary sensorymotor cortex (near area 6; Talairach et al., 1967). The center of this putative frontal eye field region is approximately $14 \mathrm{~mm}$ in vector distance from the one active in this shifting attention condition.

\section{Other visuospatial processing regions}

Another set of brain regions was inconsistently activated across conditions or only in one of the two levels of subtractions. We will discuss some of these findings because their pattern of activation or anatomical location suggests their importance in visuospatial processing.

\section{Inferior parietal/superior temporal cortex}

Responses were localized on the supramarginal gyrus in the inferior parietal lobule (IPL; area 40) during some left visual field conditions (shifting attention, passive), and on the superior temporal gyrus (STG; area 22) during right visual field conditions (shifting attention, passive, central detection). Responses were generally low in magnitude and variable in location, but they all mapped on the right hemisphere. It is our impression that these detection tasks failed to drive this region efficiently.

Given that lesions in the temporo-parietal junction produce neglect phenomena that have been related to deficits in spatial attention, one might have expected more consistent activation of this region in our study. This area, however, may mediate attentional/perceptual processes that are not emphasized in our paradigm. For example, neglect patients show larger impairment on invalid than valid trials of spatial cueing tasks (Posner et al., 1984; Baynes et al., 1986; Morrow and Ratcliff, 1988), suggesting a greater impairment in the disengage component of shifting attention. Since invalid trials were rarely presented in our paradigm, which therefore de-emphasized "disengage"-related activity, one might expect IPL/STG to be only weakly activated in the present experiment. ${ }^{2}$

Similarly, several studies indicate that lesions of IPL/STG impair the perception and selection of different scales of an object (Robertson et al., 1988; Robertson and Lamb, 1991). Robertson and colleagues have proposed that some components of unilateral neglect after right hemisphere damage can be explained by a disruption of global processing. This selection mechanism would also not be active in the current paradigm.

\section{Precuneus}

The precuneus in medial superior parietal cortex (near medial area 7) was bilaterally active (independently of the side of attention) during the shifting attention task, more consistently when the central detection condition was subtracted out. Haxby et al. (1991) reported precuneus activation ( $24 \mathrm{~mm}$ in vectorial distance from this response) during a visual dot-matching task involving several different processing components (e.g., location analysis, mental rotation).

\section{Anterior cingulate}

The anterior cingulate cortex (near area 24) was weakly activated in the subtraction between each shifting attention condition and the corresponding central detection condition. In the subtraction with the fixation point control, the anterior cingulate was not detected as an independent activation from SMA, which was powerfully activated in all conditions that included a speeded response. This response may then represent a true signal from the anterior cingulate, which was spatially masked in the subtraction with the fixation point control by a stronger adjacent SMA activation, or a differential SMA activation between shifting attention and central detection condition.

A region in the anterior cingulate cortex, similar in location to the present one, has been activated by a variety of tasks involving visual selection (Pardo et al., 1990; Corbetta et al., 1991 a), single-word processing (Petersen et al., 1988, 1989; Wise et al., 1991), and motor planning (Deiber et al., 1991). Anterior cingulate activity in all cases was coupled to prefrontal activity, and at least in the verb generate task its activation is consistently modulated by practice-related effects (Raichle et al., 1991). Although originally considered a high-level attentional center for both language and visual processing (Posner et al., 1987b; Posner and Petersen, 1990), the anterior cingulate has been recently conceptualized as part of a response selection system that is recruited when the underlying processing proceeds in a controlled manncr (as opposcd to automatic) (Corbetta et al., 1991a). It is unclear how the anterior cingulate response in the shifting attention task could be predicted by this theoretical framework.

\section{Motor regions}

Several brain regions including contralateral primary sensorimotor cortex, bilateral SMA (yet stronger contralaterally), ipsilateral superoanterior cerebellum, and contralateral inferior frontal cortex were presumably activated by the finger key press

\footnotetext{
${ }^{2}$ We thank Glyn Humphreys for this suggestion.
} 
speeded response (average RT, less than $300 \mathrm{msec}$ ). The first three regions were strictly related to the motor response, since they were absent in the passive task. The fourth region was also active in the passive task, and may be considered a visuomotor region.

Activations of primary motor, SMA, ipsilateral cerebellum, and lateral premotor cortex have been already reported in association with nonspeeded proximal and distal arm movements (Roland et al., 1980a,b; Fox et al., 1985b,c; Colebatch et al., 1991; Deiber et al., 1991; Grafton et al., 1991).

The activity in several motor-related regions was modulated by the side of stimulus presentation. Primary sensorimotor cortex and SMA activations were greater when subjects responded with their left hand to ipsilateral (left field) than contralateral (right field) stimuli (shifting attention). A similar asymmetry was present when stimuli were responded to centrally, in the presence of either ipsilateral (left field) or contralateral (right field) distractors (central detection). The effect was very robust across conditions, although of uncertain interpretation. Before concluding that the relationship reflects an effect of stimulushand laterality, it will be important to demonstrate that the asymmetry flips when the right instead of left hand is used. In addition, it will be necessary to determine if the movement characteristics of the key press response vary with the coupling of hand and visual field.

\section{Relevance for unilateral neglect}

The syndrome of unilateral neglect is characterized by an inability to attend and respond to sensory stimuli on the contralesional side, which cannot be accounted for by simple sensory or motor problems (DeRenzi, 1982). Unilateral neglect is presently considered the result of damage to spatial selection mechanisms, and is the best lesion model in humans for studying neural mechanisms of spatial behavior.

The present PET findings are relevant in several ways to the interpretation of unilateral neglect in humans. The following text will discuss three main issues: (1) unilateral neglect represents a deficit in visual orienting; (2) spatial directed attention is controlled within a distributed cortical network; (3) unilateral neglect is more frequent and profound after right hemisphere lesions.

Lesion studies indicate that patients with lesions centered in the parietal lobc, who show cxtinction (i.c., the inability to detect contralateral stimuli when they are presented simultaneously with ipsilateral stimuli), present a deficit in shifting attention toward the contralesional space when attention is engaged elsewhere (Posner et al., 1984). The deficit in covert orienting is greater for left side than for right side stimuli (Posner et al., 1984; Baynes et al., 1986; Morrow and Ratcliff, 1988; Ladavas et al., 1989) and has been correlated with the severity and degree of recovery of clinical neglect (Morrow and Ratcliffe, 1987, 1988). Interestingly, in one study the degree of visual attention impairment was correlated with the degree of anatomical damage in the superior parietal lobe (Posner et al., 1984). Some authors consider this deficit in orienting as the cognitive component critically damaged in unilateral neglect (Posner et al., 1984; Farah, 1990). As noted earlier, it is unclear if the attentional component activated in our paradigm corresponds to the sclcction mechanisms that are impaired in neglect.

It is our opinion, however, that deficits in spatial selection mechanisms cannot fully explain the wide range of observed phenomena. The syndrome of unilateral neglect after tempo- roparietal injury more likely represents a combination of deficits in selective mechanisms such as visual orienting and local/global processing, and nonspecific mechanisms, such as arousal/vigilance.

Unilateral neglect patients show blunted autonomic responses, which physiologically accompany oscillations in arousal levels, during various nonlateralized stimulation conditions. Patients with right hemisphere damage, particularly with unilateral neglect, are also slower than left hemisphere damage patients during simple nonlateralized RT tasks (Howes and Bollcr, 1975; Coslett et al., 1987). Ladavas et al. (1989) have shown that this RT slowing is common to both temporal- and parietallesioned patients, whereas only the latter group manifests selective deficits in orienting (see also Posner et al., 1987a). These findings suggest a deficit in vigilance/arousal mechanisms in unilateral neglect.

Finally, PET activation studies also suggest that nonspecific attentional mechanisms are localized in regions that produce neglect. Pardo et al. (1991) have localized a right parietofrontal system that is active during a visual vigilance task not requiring lateralized orienting. Vigilance-related responses do not overlap with the activations in superior parietal lobe for lateralized shifts of attention, or with the activation in right IPL/STG of the present study. In summary, several anatomofunctional regions are probably localized in the cortex that has been commonly associated with unilateral neglect. The syndrome may represent a variable combination of these information processing deficits.

Neural models, based on anatomical tracing and lesion evidence, have proposed that spatially oriented behavior may be controlled within a distributed brain system, including posterior parietal, prefrontal, superior temporal, and anterior cingulate cortex, and related subcortical structures (Goldman-Rakic, 1988). One important issue dealing with distributed neural systems is whether all components in the system are activated by any task processed within the networks, or whether individual components are activated as a function of task demands.

In the present experiment, the superior parietal region was activated by voluntary or automatic shifts of attention independent of the execution of an overt response, while activation of the superior frontal region depended on the actual execution of a motor response. The recruitment of each region was therefore controlled by specific task demands, and not automatic across tasks. Lesion studics support this view. Posterior parietal lesions seem to be associated with deficits that are "perceptual" in nature, such as the inability to detect a sensory stimulus on the contralesional side when presented along with another stimulus on the ipsilesional side (extinction; Daffner et al., 1990), or the inability to judge the midline of a rod (Bisiach et al., 1990). Frontal lesions seem to be associated with deficits that are more "premotor" in nature, such as a difficulty in initiating and/or performing directional arm movements toward the contralesional side of space, independent of the side of stimulus presentation (Heilman et al., 1985; Bisiach et al., 1990; Coslett et al., 1990). According to Mesulam (1981, 1990), posterior parietal cortex provides a sensory representation of extrapersonal space, and frontal cortex provides a map for the distribution of orienting and exploratory movements. Both PET and lesion evidence, therefore, support computational models where functional specialization is maintaincd at the regional level, and not distributed in the whole network.

Finally, several theories have been proposed to account for the higher frequency of unilateral neglect after right than left 
hemisphere lesions. One theory, hased on a hemispheric directional bias for attention (Kinsbourne, 1977, 1987), is inconsistent with the absence of any directional effects on blood flow responses. Another set of theories postulates that unilateral neglect represents a deficit of attentional and/or intentional mechanisms that are organized in hemifield/hemispace coordinates (Mesulam, 1981; Heilman et al., 1987a). The asymmetry arises because the right hemisphere is able to direct attention in both visual fields, while the left hemisphere can only control attention to the right visual field. Our PET findings suggest that there are two distinct representations in the right superior parietal lobule for separately directing attention into the left or right visual field, and one representation in the left superior parietal lobule for directing attention mainly into the right visual field. This asymmetry may explain the greater "disengage" deficits found in spatial cueing tasks following right than left parietal lesions, but cannot account for the higher incidence of arousal/vigilance deficits following right hemisphere lesions.

\section{Conclusions}

Shifts of visuospatial attention during lateralized luminance detection tasks selectively activated regions of the superior parietal and frontal cortex. Both superior parietal and frontal activations encoded the visual field rather than the direction of an attention shift. Furthermore, in the right hemisphere superior parietal responses for selection of left and right field were spatially segregated. Finally, superior parietal and frontal regions were related to different aspects of spatial selection. The parietal region was active when attention was shifted on the basis of cognitive or sensory cues, independent of the execution of an overt response. The frontal region was active only when sclcctcd latcralized stimuli were overtly detected. These findings indicate that parietal and frontal cortex differentially contribute to the cortical control of spatial selection.

\section{References}

Andersen RA (1989) Visual and eye movement functions of the posterior parietal cortex. Annu Rev Neurosci 12:377-403.

Andersen RA, Essick GK, Siegel RM (1985) Encoding of spatial location by posterior parietal neurons. Science 239:456-458.

Andersen RA, Asanuma C, Essick G, Siegel RM (1990a) Corticocortical connections of anatomically and physiologically defined subdivisions within the inferior parietal lobule. J Comp Neurol 296:65113.

Andersen RA, Bracewell RM, Barash S, Gnadt JW, Fogassi L (1990b) Eye position effects on visual, memory, and saccade-related activity in areas LIP and 7A of macaque. J Neurosci 10:1176-1196.

Baynes K, Holtzman JD, Volpe BT (1986) Components of visual attention: alterations in response pattern to visual stimuli following parietal lobe infarction. Brain 109:99-114.

Berlucchi G, Tassinari G, Marzi CA, Di Stefano M (1989) Spatial distribution of the inhibitory effects of peripheral non-informative cues on simple reaction time to non-fixated visual targets. Neuropsychologia 27:201-221.

Bisiach E, Luzzatti C, Perani D (1981) Unilateral neglect, representational schema and consciousness. Brain 102:757-765.

Bisiach E, Geminiani G, Berti A, Rusconi ML (1990) Perceptual and premotor factors of unilateral neglect. Neurology 40:1278-1281.

Boch RA, Goldberg ME (1989) Participation of prefrontal neurons in the preparation of visually guided eye movements in the rhesus monkey. J Neurophysiol 61:1064-1084.

Brodmann K (1909) Vergleichende lokalisationlehre der grosshirnrinde in inren prinzipien dargestellt auf grund des zellenbaues. Leipzig: Barth.

Bruce CJ, Goldberg ME (1985) Primate frontal eye fields. I. Single neurons discharging before saccades. J Neurophysiol 53:603-635.

Bushnell MC, Goldberg ME, Robinson DL (1981) Behavioral en- hancement of visual responses in monkey cerebral cortex. I. Modulation in posterior parietal cortex related to selective attention. J Neurophysiol 46:755-772.

Calvanio R, Petrone PN, Levine DN (1987) Left visual spatial neglect is both environment-centered and body-centered. Neurology 37:11791183.

Colby CL (1991) The neuroanatomy and neurophysiology of attention. J Child Neurol 6:S90-S118.

Colby CL, Duhamel J (1991) Heterogeneity of extrastriate visual areas and multiple parietal areas in the macaque monkey. Neuropsychologia 29:517-537.

Colebatch JG, Deiber M-P, Passingham RE, Friston KJ, Frackowiak RSJ (1991) Regional cerebral blood flow during voluntary arm and hand movements in human subjects. J Neurophysiol 65:1392-1401.

Corbetta M, Miezin FM, Dobmeyer S, Shulman GI, Petersen SE. (1990) Attentional modulation of neural processing of shape, color, and velocity in humans. Science 248:1556-1559.

Corbetta M, Miezin FM, Dobmeyer S, Shulman GL, Petersen SE (1991a) Selective and divided attention during visual discrimination of shape, color, and speed: functional anatomy by positron emission tomography. J Neurusci 11:2383-2402.

Corbetta M, Miezin FM, Shulman GL, Petersen SE (1991b) Selective attention modulates extrastriate visual regions in humans during visual feature discrimination and recognition. In: Ciba Foundation Symposium 163, Exploring brain functional anatomy with positron tomography (Chadwick DJ, Whelan J, eds), pp. 165-180. Chichester: Wiley.

Coslett HB, Bowers D, Heilman KM (1987) Reduction in cerebral activation after right hemisphere stroke. Neurolngy 37:957-962.

Coslett HB, Bowers D, Fitzpatrick E, Haws B, Heilman KM (1990) Directional hypokinesia and hemispatial inattention in neglect. Brain 113:475-486.

Daffner KR, Ahern GL, Weintraub S, Mesulam M-M (1990) Dissociated neglect behavior following sequential strokes in the right hemisphere. Ann Neurol 28:97-100.

Deiber M-P, Passingham RE, Colebatch JG, Friston KJ, Nixon PD, Frackowiak RSJ (1991) Cortical areas and the selection of movement: a study with positron emission tomography. Exp Brain Res 84: $393-402$.

DeRenzi E (1982) Disorders of space exploration and cognition. New York: Wiley.

Deuel RM, Collins RC (1984) The functional anatomy of frontal lobe neglect in the monkey: behavioral and quantitative 2-deoxyglucose studies. Ann Neurol 15:521-529.

Downing CJ (1988) Expectancy and visual-spatial attention effects on vision. J Exp Psychol [Hum Percept] 14:188-197.

Duhamel J, Colby CL, Goldberg ME (1992) The updating of the representation of visual space in parietal cortex by intended eyemovements. Science 255:90-92.

Eidelberg D, Galaburda AM (1984) Inferior parietal lobule. Divergent architectonic asymmetries in the human brain. Arch Neurol 41:843852.

Eriksen CW, Hoffman JE (1972) Temporal and spatial characteristics of selective encoding from visual displays. Percept Psychophys 12: 201-204.

Eriksen CW, Yeh Y (1985) Allocation of attention in the visual field. J Fxp Psychol [Hum Percept] 11:583-597.

Farah MJ (1990) Visual agnosia. Cambridge: MIT Press

Farah MJ, Brunn JL, Wong AB, Wallace MA, Carpenter PA (1990) Frames of reference for allocating attention to space: evidence from the neglect syndrome. Neuropsychologia 28:335-347.

Fox PT, Mintun MA (1989) Noninvasive functional brain mapping by change-distribution analysis of averaged PET images of $\mathrm{H}_{2}{ }^{15} \mathrm{O}$. J Nucl Med 30:141-149.

Fox PT, Perlmutter JS, Raichle ME (1985a) A stereotactic method of anatomical localization for positron emission tomography. J Comput Assist Tomogr 9:141-153.

Fox PT, Raichle ME, Thach WT (1985b) Functional mapping of the human cerebellum with positron emission tomography. Proc Natl Acad Sci USA 82:7462-7466.

Fox PT, Fox JM, Raichle ME, Burde RM (1985c) The role of cerebral cortex in the generation of voluntary saccades: a positron emission tomographic study. J Neurophysiol 54:348-369.

Fox PT, Miezin FM, Allman JM, Van Essen DC, Raichle ME (1987) Retinotopic organization of human visual cortex mapped with positron emission tomography. J Neurosci 7:913-922. 
Fox PT, Mintun MA, Reiman EM, Raichle ME (1988) Enhanced detection of focal brain responses using intersubject averaging and change-distribution analysis of subtracted PET images. J Cereb Blood Flow Metab 8:642-653.

Friston KJ, Frith CD, Liddle PF, Frackowiack RSJ (1991) Comparing functional (PET) images: the assessment of significant change. J Cereb Blood Flow Metab 11:690-699.

Frith CD, Friston K, Liddle PF, Frackowiak RSJ (1991) Willed action and the prefrontal cortex in man: a study with PET. Proc R Soc Lond [Biol] 244:241-246.

Funahashi S, Bruce CJ, Goldman-Rakic PS (1991) Neuronal activity related to saccadic eye movements in the monkey's dorsolateral prefrontal cortex. J Neurophysiol 65:1464-1483.

Goldman-Rakic PS (1987) Circuitry of primate prefrontal cortex and regulation of behavior by representational knowledge. In: The handbook of physiology, Sec 1, The nervous system, Vol V, Higher functions of the brain, Pt 1 (Plum F, Mountcastle V, eds), pp 373-417. Bethesda, MD: American Physiological Society.

Goldman-Rakic PS (1988) Topography of cognition: parallel distributed networks in primate association cortex. Annu Rev Neurosci 11: $137-156$.

Grafton ST, Woods RP, Mazziotta JC, Phelps ME (1991) Somatotopic mapping of the primary motor cortex in humans: activation studies with cerebral blood flow and positron emission tomography. J Neurophysiol 66:735-743.

Harter MR, Aine C, Schroeder C (1982) Hemispheric differences in the neural processing of stimulus location and type: effects of selective attention on visual evoked potentials. Neuropsychologia 20:421-438.

Haxby JV, Grady CL, Horwitz B, Ungerleider LG, Mishkin M, Carson KE, Herscovitch P, Schapiro MB, Rapoport SI (1991) Dissociation of object and spatial visual processing pathways in human extrastriate cortex. Proc Natl Acad Sci USA 88:1621-1625.

Heilman KM, Valenstein E (1972) Frontal lobe neglect in man. Neurology 22:660-664.

Heilman KM, Bowers D, Coslett HB, Whelan H, Watson RT (1985) Directional hypokinesia: prolonged reaction times for leftward movements in patients with right hemisphere lesions and neglect. Neurology 35:855-859.

Heilman KM, Watson RT, Valenstein E, Goldberg ME (1987a) Attention: behaviour and neural mechanisms. In: The handbook of physiology, Sect 1, The nervous system, Vol V, Higher functions of the brain, Pt 2 (Plum F, Mountcastle VB, Geiger ST, eds), pp 461481. Bethesda, MD: American Physiological Society.

Heilman KM, Bowers D, Valenstein E, Watson RT (1987b) Hemispace and hemispatial neglect. In: Neurophysiological and neuropsychological aspects of spatial neglect (Jeannerod M, ed), pp 115-150. Amsterdam: North-Holland-Elsevier.

Herscovitch P, Markham J, Raichle ME (1983) Brain blood flow measured with intravenous $\mathrm{H}_{2}{ }^{15} \mathrm{O}$. I. Theory and error analysis. J Nucl Med 24:782-789.

Hikosaka O, Sakamoto M, Usui S (1989) Functional properties of monkey caudate neurons. I. Activities related to saccadic eye movements. J Neurophysiol 61:780-798.

Hillyard SA, Munte TF (1984) Selective attention to color and localion: an analysis with event-related brain potentials. Percept Psychophys 36:185-198.

Hillyard SA, Picton TW (1987) Electrophysiology of cognition. In: The handbook of physiology, Sect 1 , The nervous system, Vol V, Higher functions of the brain, Pt 2 (Plum F, Mountcastle VB, Geiger ST, eds), pp 519-584. Bethesda, MD: American Physiological Society.

Howes D, Boller F (1975) Simple reaction time: evidence for focal impairment from lesions of the right hemisphere. Brain 98:317-332.

Hughes HC, Zimba LD (1987) Natural boundaries for the spatial spread of directed visual attention. Neuropsychologia 25:5-18.

Hyvarinen $H$ (1981) Regional distribution of functions in parietal association area 7 of the monkey. Brain Res 206:287-303.

Jones EG, Powell TPS (1970) An anatomical study of converging sensory pathways within the cerebral cortex of the monkey. Brain 93: $793-820$

Jonides J (1981) Voluntary vs. automatic control over the mind's eye's movement. In: Attention and performance, Vol XI (Posner MI, Marin O, eds), pp 187-205. Hillsdale, NJ: Erlbaum.

Kennard MA (1939) Alterations in response to visual stimuli following lesions of frontal lobe in monkeys. Arch Neurol Psychiatry 41:11531165 .

Kinsbourne M (1977) Hemi-neglect and hemisphere rivalry. In: Hemi- inattention and hemispheric specialization (Weinstein EA, Friedland RL, eds), pp 41-52. New York: Raven.

Kinsbourne M (1987) Mechanisms of unilateral neglect. In: Neurophysiological and neuropsychological aspects of spatial neglect (Jeannerod M, ed), pp 69-86. Amsterdam: Elsevier.

LaBerge D, Brown V (1989) Theory of attentional operations in shape identification. Psychol Rev 96:101-124.

LaBerge D, Buchsbaum MS (1990) Positron emission tomographic measurements of pulvinar activity during an attention task. J Neurosci 10:613-619.

Ladavas E (1987) Is hemispatial deficit produced by right parietal lobe damage associated with retinal or gravitational coordinates? Brain 110:167-180.

Ladavas E, Del Pesce M, Provinciali L (1989) Unilateral attention deficits and hemispheric asymmetries in the control of visual attention. Neuropsychologia 27:353-366.

Latto R, Cowey A (1971) Visual field defects after frontal eye-field lesions in monkeys. Brain Res 30:1-24.

Mangun GR, Hansen JC, Hillyard SA (1987) The spatial orienting of attention: sensory facilitation or response bias? Electroencephalogr Clin Physiol [Suppl] 40:118-124.

Mangun GR, Hillyard SA, Luck SJ (1992) Electrocortical substrates of visual selective attention. In: Attention and performance XIV (Meyer D, Kornblum S, eds). Hillsdale, NJ: Erlbaum.

Matelli M, Camarda R, Glickstein M, Rizzolatti G (1986) Afferent and efferent projections of the inferior area 6 in the macaque monkey. J Comp Neurol 251:281-298.

Maylor EA (1985) Facilitory and inhibitory components of orienting in visual space. In: Attention and performance XI (Posner MI, Marin, OSM, eds), pp 89-104. Hillsdale, NJ: Erlbaum.

Mesulam MM (1981) A cortical network for directed attention and unilateral neglect. Ann Neurol 10:309-315.

Mesulam MM (1990) Large-scale neurocognitive networks and distributed processing for attention, language, and memory. Ann Neurol 28:597-613

Milner AD (1987) Animal models for the syndrome of spatial neglect. In: Neurophysiological and neuropsychological aspects of spatial neglect (Jeannerod M, ed), pp 259-288. Amsterdam: North-HollandElsevier.

Mintun MA, Fox PT, Raichle ME (1989) A highly accurate method of localizing regions of neuronal activation in the human brain with positron emission tomography. J Cereb Blood Flow Metab 9:96-103.

Morrow LA, Ratcliff G (1987) Attentional mechanisms in clinical neglect. J Clin Exp Neuropsychol 9:74-75.

Morrow LA, Ratcliff G (1988) The disengagement of covert attention and the neglect syndrome. Psychobiology 16:261-269.

Muller HJ, Rabbit MA (1989) Reflexive and voluntary orienting of visual attention: time course of activation and resistance to interruption. J Exp Psychol [Gen] 15:315-330.

Pardo JV, Pardo PJ, Janer KW, Raichle ME (1990) The anterior cingular cortex mediates processing selection in the Stroop attentional conflict paradigm. Proc Natl Acad Sci USA 87:256-259.

Pardo JV, Fox PT, Raichle ME (1991) Localization of a human system for sustained attention by positron emission tomography. Nature 349 : 61-64.

Passingham RE (1988) Premotor cortex and preparation for movement. Exp Brain Res 70:590-596.

Petersen SE, Robinson DL, Keys W (1985) Pulvinar nuclei of the behaving rhesus monkey: visual responses and their modulation. $J$ Neurophysiol 54:867-886.

Petersen SE, Robinson DL, Morris JD (1987) Contributions of the pulvinar to visual spatial attention. Neuropsychologia 25:97-105.

Petersen SE, Fox PT, Posner MI, Mintun M, Raichle ME (1988) Positron emission tomographic studies of the cortical anatomy of singleword processing. Nature 331:585-589.

Petersen SE, Fox PT, Posner MI, Mintun M, Raichle ME (1989) Positron emission tomographic studies of the processing of single words. J Cogn Neurosci 1:153-170.

Petersen SE, Corbetta M, Miezin FM, Shulman GL, Raichle ME (in press) The effects of selective attention on visual processing measured with performance and PET (positron emission tomography). In: Brain mechanisms of perception and memory: from neuron to behavior (Ono T, Squire L, Perrett D, Raichle ME, eds), in press. New York: Oxford UP.

Petrides M, Pandya DN (1984) Projections to the frontal cortex from 
the posterior parietal region in the Rhesus monkey. J Comp Neurol 228:105-116.

Posner MI (1980) Orienting of attention. Q J Exp Psychol 32:3-25.

Posner MI, Cohen Y (1984) Components of performance. In: Attention and performance (Bouma $\mathrm{H}$, Bowhuis D, eds), pp 531-556. Hillsdale, NJ: Erlbaum.

Posner MI, Petersen SE (1990) The attention system of the human brain. Annu Rev Neurosci 13:25-42.

Posner MI, Cohen Y, Rafal RD (1982) Neural systems control over spatial orienting. Philos Trans R Soc Lond [Biol] 2908:187-198.

Posner MI, Walker JA, Friedrich FJ, Rafal RD (1984) Effects of parietal injury on covert orienting of visual attention. J Neurosci 4:18631874.

Posner MI, Walker JA, Friedrich FJ, Rafal RD (1987a) How do the parietal lobes direct covert attention? Neuropsychologia 25:135-145.

Posner MI, Inhoff A, Friedrich FJ, Cohen A (1987b) Isolating attentional systems: a cognitive-anatomical analysis. Psychobiology 15: 107-121.

Rafal RD, Posner MI (1987) Deficits in human visual spatial attention following thalamic lesions. Proc Natl Acad Sci USA 84:7349-7353.

Raichle ME, Fiez J, Videen TO, Fox PT, Pardo JV, Petersen SE (1991) Practice-related changes in human brain functional anatomy. Soc Neurosci Abstr 17:21.

Remington R (1980) Attention and saccadic eye movements. J Exp Psychol [Hum Percept] 6:726-744.

Reuter-Lorenz PA, Posner MI (1990) Components of neglect from right-hemisphere damage: an analysis of line bisection. Neuropsychologia 28:327-333.

Richmond BJ, Wurtz RH, Sato T (1983) Visual responses of inferior temporal neurons in the awake rhesus monkey. J Neurophysiol 50: 1415-1432.

Rizzolatti G, Camarda R (1987) Neural circuits for spatial attention and unilateral neglect. In: Neurophysiological and neuropsychological aspects of spatial neglect (Jeannerod M, ed), pp 289-313. Amsterdam: North-Holland-Elsevier.

Rizzolatti G, Matelli M, Pavesi G (1983) Deficits in attention and movement following the removal of postarcuate (area 6) and prearcuate (area 8) cortex in macaque monkeys. Brain 106:655-673.

Rizzolatti G, Riggio L, Dascola I, Umilta C (1987) Reorienting attention across the horizontal and vertical meridians: evidence in favor of a premotor theory of attention. Neuropsychologia 25:31-40.

Robertson LC, Lamb M (1991) Neuropsychological contributions to theories of part/whole organization. Cogn Psychol 23:293-330.

Robertson LC, Lamb MR, Knight RT (1988) Effects of lesions of temporal-parietal junction on perceptual and attentional processing in humans. J Neurosci 8:3757-3769.

Robinson DL, Currie J, Petersen SE (1985) Effects of parietal damage on attentional behavior. Invest Ophthalmol Vis Sci [Suppl] 26:266.

Robinson DL, Bowman EM, Kertzman C (1991) Covert orienting of attention in macaque: II. A signal in parietal cortex to disengage attention. Soc Neurosci Abstr 17:442.

Roland PE, Friberg L (1985) Localization of cortical areas activated by thinking. J Neurophysiol 53:1219-1243.

Roland P, Larson B, Lassen NA, Skinhøj E (1980a) Supplementary motor area and other areas in organization of voluntary movements in man. J Neurophysiol 43:118-136.
Roland PE, Skinhøj E, Lassen NA, Larsen B (1980b) Different cortical areas in man in organization of voluntary movements in extrapersonal space. J Neurophysiol 43:137-150.

Sato T (1989) Interactions of visual stimuli in the receptive fields of inferior temporal neurons in awake macaques. J Neurophysiol 77: 23-30.

Selemon LD, Goldman-Rakic PS (1988) Common cortical and subcortical target areas of the dorsolateral prefrontal and posterior parietal cortices in the rhesus monkey: evidence for a distributed neural network subserving spatial guided behavior. J Neurosci 8:4049-4068.

Shepherd M, Findlay JM, Hockey RJ (1986) The relationship between eye movements and spatial attention. Q J Exp Psychol 38:475-491.

Shulman GL, Wilson J, Sheehy JB (1985) Spatial determinants of the distribution of attention. Percept Psychophys 37:59-65.

Squire LR, Ojemann JG, Miezin FM, Petersen SE, Videen TO, Raichle ME (1991) Activation of the hippocampus in normal humans: a functional anatomical study of memory. Proc Natl Acad Sci USA 89: 1837-1841

Talairach J, Szikla G, Tournoux P (1967) Atlas d'anatomie stereotaxique du telencephale. Paris: Masson.

Tassinari G, Aglioti S, Chelazzi L, Marzi CA, Berlucchi G (1987) Distribution in the visual field of the costs of voluntarily allocated attention and of the inhibitory after-effects of covert orienting. Neuropsychologia 25:55-71.

Ullman S (1984) Visual routines. Cognition 18:97-159.

Ungerleider LG, Desimone R (1986) Cortical connections of visual area $\mathrm{MT}$ in the macaque. J Comp Neurol 248:190-222.

Videen TO, Snyder AZ, Raichle ME (1991) Optimization of regionof-interest definition for detecting regional $\mathrm{CBF}$ activation with positron emission tomography. J Cereb Blood Flow Metab 11:S571.

Von Bonin G, Bailey P (1947) The neocortex of macaca mulatta. Urbana: University Illinois.

Wise R, Hadar U, Howard D, Patterson K (1991) Language activation studies with positron emission tomography. In: Ciba Foundation Symposium 163, Exploring brain functional anatomy with positron emission tomography, pp. 218-234. Chichester: Wiley.

Wise SP (1987) Functional fractionation of frontal fields. Trends Neurosci 10:449-451.

Worsley KJ, Evans AC, Marrett S, Neelin P (1992) A three dimensional statistical analysis for CBF activation studies in human brain. J Cereb Blood Flow Metab 12:900-918.

Wurtz RH, Goldberg ME (1972) Activity of superior colliculus in behaving monkey. II. Effect of attention on neuronal responses. J Neurophysiol 35:560-574.

Wurtz RH, Mohler CW (1976) Organization of monkey superior colliculus: enhanced visual response of superficial layer cells. J Neurophysiol 39:745-765.

Yamamoto M, Ficke DC, Ter-Pogossian M (1982) Performance study of PETT VI, a positron computed tomograph with 288 cesium fluoride detectors. IEEE Trans Nucl Sci 29:529-533.

Yantis S, Jonides J (1990) Abrupt visual onsets and selective attention: voluntary versus automatic allocation. J Exp Psychol [Hum Percept] 16:121-134. 